The cumulant process and Esscher's change of measure

Kallsen, Jan and Shiryaev, Albert N.

2002

MIMS EPrint: 2007.175

Manchester Institute for Mathematical Sciences

School of Mathematics

The University of Manchester

\footnotetext{
Reports available from: http://eprints.maths.manchester.ac.uk/

And by contacting: The MIMS Secretary

School of Mathematics

The University of Manchester

Manchester, M13 9PL, UK
} 


\title{
The cumulant process and Esscher's change of measure
}

\author{
Jan Kallsen ${ }^{1}$, Albert N. Shiryaev ${ }^{2}$ \\ ${ }^{1}$ Institut für Mathematische Stochastik, Universität Freiburg, Eckerstraße 1, 79104 Freiburg i. Br., \\ Germany (e-mail: kallsen@ stochastik.uni-freiburg.de) \\ 2 Steklov Mathematical Institute, Gubkina St. 8, 117966 Moscow, Russia (e-mail: shiryaev@mi.ras.ru)
}

\begin{abstract}
In this paper two kinds of cumulant processes are studied in a general setting. These processes generalize the cumulant of an infinitely divisible random variable and they appear as the exponential compensator of a semimartingale. In a financial context cumulant processes lead to a generalized Esscher transform. We also provide some new criteria for uniform integrability of exponential martingales.

Key words: Cumulant process, stochastic logarithm, exponential transform, exponential compensator, exponentially special semimartingale, Esscher transform, uniform integrability
\end{abstract}

JEL Classification: G13, D52

Mathematics Subject Classification (2000): 60G48, 91B24

\section{Introduction}

Any exponential semimartingale, i.e. any process of the form $S=\exp (X)$ for some semimartingale $X$, can be written as stochastic exponential $S=\mathcal{E}(\widetilde{X})$ for some semimartingale $\widetilde{X}$. The process $\widetilde{X}$ is called stochastic logarithm of $S$ or exponential transform of $X$. If $\widetilde{X}$ is a special semimartingale, then its predictable part of finite variation $\widetilde{K}$ will be denoted the Laplace cumulant process of $X$. This process is closely linked to the exponential compensator of $X$, i.e. the unique predictable process $K$ of finite variation such that $\exp (X-K)$ is a local martingale. In fact, $\widetilde{K}$ is the exponential transform of $K$. These notions and their properties are the subject of the subsequent section. 
The question of uniform integrability of local martingales $\exp (X-K)$ plays an important role in statistics and finance because they appear as candidates for density processes. Among others, Gihman and Skorohod (1972), Grigelionis (1971), Liptser and Shiryaev (1972), Novikov (1972, 1975, 1979, 1980), Jacod and Mémin (1976), Kazamaki (1977, 1978a,b, 1979), Mémin (1978), Lépingle and Mémin (1978a,b), Mémin and Shiryaev (1979), Kabanov et al. (1979, 1980), Okada (1982), Yan (1980, 1982a,b), Kazamaki and Sekiguchi (1982, 1983), Stummer (1993), Jerschow (1994), Kramkov and Shiryaev (1998) contributed to this issue. In Sect. 3, we show that the cumulant process naturally leads to a hierachy of sufficient criterions for uniform integrability of $\exp (X-K)$, which includes in particular Novikovand Kazamaki-type conditions in the sense of Revuz and Yor (1999), Propositions VIII.1.14 and VIII.1.15.

In mathematical finance, measure transformations with density process $\left.\frac{d P_{\vartheta}}{d P}\right|_{\mathcal{F}_{t}}$ $=\exp \left(\vartheta X_{t}-k(\vartheta) t\right)$ for some given Lévy process $X$ and real numbers $\vartheta, k(\vartheta)$ have been considered under the name Esscher transform for contingent claim pricing (cf., e.g., Gerber and Shiu 1994; Madan and Milne 1991; Eberlein and Keller 1995; Chan 1999). This concept can be generalized to integrals $\int_{0}^{\cdot} \vartheta_{s} d X_{s}$ of a large class of semimartingales if the cumulant $k(\vartheta) t$ is replaced with the cumulant process of $\int_{0}^{\cdot} \vartheta_{s} d X_{s}$. This approach is discussed in Sect. 4.

We generally use the notation of Jacod and Shiryaev (1987) (henceforth JS) and Jacod $(1979,1980)$. The transposed of a vector or matrix $x$ is denoted as $x^{\top}$ and its components by superscripts. In particular, $\vartheta^{\top} \cdot X$ denotes the stochastic integral of $\vartheta$ relative to $X$. Increasing processes are identified with their corresponding Lebesgue-Stieltjes measure.

\section{Cumulant processes}

\subsection{Stochastic exponential and logarithm}

In this section we review and introduce some notions of stochastic calculus that turn out to be useful for the study of exponential semimartingales. Firstly, we recall the definition of the stochastic or Doléans-Dade exponential of a semimartingale.

Definition 2.1 Let $X$ be a real-valued semimartingale. The stochastic exponential $\mathcal{E}(X)$ is defined as the (up to indistinguishability unique) solution $Z$ to the stochastic differential equation

$$
Z=1+Z_{-} \cdot X
$$

The mapping $X \mapsto \mathcal{E}(X)$ can be inverted. In analogy to real calculus, we call its converse the stochastic logarithm of $X$ (cf., also Foldes 1990; Choulli et al. 1998; Kallsen and Shiryaev 2000).

Lemma 2.2 Let $Z$ be a semimartingale such that $Z, Z_{-}$are $\mathbb{R} \backslash\{0\}$-valued. Then there exists an up to indistinguishability unique semimartingale $X$ with $X_{0}=0$ and $Z=Z_{0} \mathcal{E}(X)$. It is given by

$$
X=\frac{1}{Z_{-}} \cdot Z
$$


Proof W.l.o.g. $Z_{0}=1$. The existence and explicit form of $X$ follows from $1+Z_{-}$. $\left(\frac{1}{Z_{-}} \cdot Z\right)=1+\left(Z_{-} \frac{1}{Z_{-}}\right) \cdot Z=Z$. Now let $X$ be any semimartingale with $X_{0}=0$ and $Z=\mathcal{E}(X)$. Then $X=\left(\frac{1}{Z_{-}} Z_{-}\right) \cdot X=\frac{1}{Z_{-}} \cdot\left(Z_{-} \cdot X\right)=\frac{1}{Z_{-}} \cdot Z$, which yields the uniqueness.

Definition 2.3 We call the process $X$ in the previous lemma stochastic logarithm of $Z$ and write $\mathcal{L}(Z):=X$.

The following lemma summarizes some properties of these processes.

Lemma 2.4 Let $\widetilde{X}, Z$ be real-valued semimartingales such that $Z, Z_{-}$are $\mathbb{R} \backslash\{0\}$ valued. Then the following statements hold.

1. $\mathcal{E}(\widetilde{X})=\exp \left(\widetilde{X}-\widetilde{X}_{0}-\frac{1}{2}\left\langle\widetilde{X}^{c}, \widetilde{X}^{c}\right\rangle\right) \prod_{s \leq .}\left(1+\Delta \widetilde{X}_{s}\right) e^{-\Delta \widetilde{X}_{s}}$

2. $\mathcal{L}(Z)=\log \left(\left|\frac{Z}{Z_{0}}\right|\right)+\frac{1}{2 Z_{-}^{2}} \cdot\left\langle Z^{c}, Z^{c}\right\rangle-\sum_{s \leq}\left(\log \left(\left|\frac{Z_{s}}{Z_{s-}}\right|\right)+1-\frac{Z_{s}}{Z_{s-}}\right)$

3. If $\Delta \widetilde{X}>-1$, then $\mathcal{E}(\widetilde{X})=\exp \left(\widetilde{X}-\widetilde{X}_{0}-\frac{1}{2}\left\langle\widetilde{X}^{c}, \widetilde{X}^{c}\right\rangle+(\log (1+x)-x) * \mu^{\tilde{X}}\right)$.

4. $\mathcal{L}(Z)=\log \left(\left|\frac{Z}{Z_{0}}\right|\right)+\frac{1}{2 Z_{-}^{2}} \cdot\left\langle Z^{c}, Z^{c}\right\rangle-\left(\log \left(\left|1+\frac{x}{Z_{-}}\right|\right)-\frac{x}{Z_{-}}\right) * \mu^{Z}$

5. If $\Delta \widetilde{X} \neq-1$ outside some evanescent set, then $\mathcal{L}(\mathcal{E}(\widetilde{X}))=\widetilde{X}-\widetilde{X}_{0}$.

6. $\mathcal{E}(\mathcal{L}(Z))=\frac{Z}{Z_{0}}$

Proof

1. JS, I.4.64

2. By Itô's formula (cf., JS, I.4.57 and Goll and Kallsen 2000, Lemma A.5), we have $\log (|Z|)=\log \left(\left|Z_{0}\right|\right)+\frac{1}{Z_{-}} \cdot Z-\frac{1}{2 Z_{-}^{2}} \cdot\left\langle Z^{c}, Z^{c}\right\rangle+\sum_{s \leq .}\left(\log \left(\left|Z_{s}\right|\right)-\right.$ $\left.\log \left(\left|Z_{s-}\right|\right)-\frac{1}{Z_{s-}} \Delta Z_{s}\right)$, which yields the assertion.

3. This follows from Statement 1 .

4. This follows from Statement 2.

5. For $Z:=\mathcal{E}(\widetilde{X})$ we have $\mathcal{L}(Z)=\frac{1}{Z_{-}} \cdot Z=\frac{1}{Z_{-}} \cdot\left(1+Z_{-} \cdot \widetilde{X}\right)=\widetilde{X}-\widetilde{X}_{0}$.

6. This holds by definition.

For the study of exponentials of semimartingales the following notions will prove useful.

Definition 2.5 For any real-valued semimartingale $X$ with $X_{0}=0$, we call $\widetilde{X}:=$ $\mathcal{L}(\exp (X))$ the exponential transform of $X$. Conversely, we call $X:=\log (\mathcal{E}(\widetilde{X}))$ the logarithmic transform of any real-valued semimartingale $\widetilde{X}$ with $\widetilde{X}_{0}=0$ and $\Delta \widetilde{X}>-1$.

Exponential and logarithmic transforms can be determined explicitly:

Lemma 2.6 Let $X$ be a real-valued semimartingale with $X_{0}=0$ and let $\widetilde{X}:=$ $\mathcal{L}(\exp (X))$ be its exponential transform (i.e., $X$ is the logarithmic transform of $\widetilde{X})$. Then we have:
1. $\tilde{X}=X+\frac{1}{2}\left\langle X^{c}, X^{c}\right\rangle+\left(e^{x}-1-x\right) * \mu^{X}$
2. $X=\widetilde{X}-\frac{1}{2}\left\langle\widetilde{X}^{c}, \widetilde{X}^{c}\right\rangle+(\log (1+x)-x) * \mu^{\widetilde{X}}$
3. $\Delta \widetilde{X}=e^{\Delta X}-1$ 
4. $\Delta X=\log (1+\Delta \widetilde{X})$

Proof

1. Note that $\Delta Z=Z_{-}\left(e^{\Delta X}-1\right)$ for $Z:=\exp (X)$ and hence $\left(\log \left(\left|1+\frac{x}{Z_{-}}\right|\right)-\right.$ $\left.\frac{x}{Z_{-}}\right) * \mu^{Z}=\left(x-e^{x}+1\right) * \mu^{X}$. From Itô's formula, it follows that $Z^{c}=e^{X_{-}} \cdot X^{c}$ (cf., e.g., the proof of Corollary A.6 in Goll and Kallsen (2000)), which implies that $\frac{1}{Z_{-}^{2}} \cdot\left\langle Z^{c}, Z^{c}\right\rangle=\left\langle X^{c}, X^{c}\right\rangle$. The claim follows now from Statement 4 in Lemma 2.4 .

2. This follows directly from Statement 3 in Lemma 2.4.

3. This is implied by Statement 1 .

4. This is implied by Statement 2 .

For some processes these transforms are of a simple form:

\section{Lemma 2.7}

1. If $X \in \mathcal{V}$ is continuous, then its exponential and logarithmic transforms coincide with $X$.

2. If $X$ is a real-valued Lévy process with characteristic triplet $(b, c, F)$ relative to some truncation function $h: \mathbb{R} \rightarrow \mathbb{R}$, then its exponential transform $\widetilde{X}$ is again a Lévy process. Its characteristic triplet $(\widetilde{b}, \widetilde{c}, \widetilde{F})$ is given by

$$
\begin{aligned}
\widetilde{b} & =b+\frac{c}{2}+\int\left(h\left(e^{x}-1\right)-h(x)\right) F(d x), \\
\widetilde{c} & =c, \\
\widetilde{F}(G) & =\int 1_{G}\left(e^{x}-1\right) F(d x) \text { for } G \in \mathcal{B} .
\end{aligned}
$$

Conversely, the logarithmic transform $X$ of a Lévy process $\tilde{X}$ with $\Delta \widetilde{X}>-1$ and characteristic triplet $(\widetilde{b}, \widetilde{c}, \widetilde{F})$ is again a Lévy process, with triplet $(b, c, F)$ as follows:

$$
\begin{aligned}
b & =\widetilde{b}-\frac{\widetilde{c}}{2}+\int(h(\log (1+x))-h(x)) \widetilde{F}(d x), \\
c & =\widetilde{c}, \\
F(G) & =\int 1_{G}(\log (1+x)) \widetilde{F}(d x) \text { for } G \in \mathcal{B} .
\end{aligned}
$$

(By Lévy process we refer to a PIIS in the sense of JS, II.4.1, II.4.19.)

Proof

1. This follows immediately from Lemma 2.6 because $X^{c}$ and $\mu^{X}$ vanish in this case.

2. Goll and Kallsen (2000), Lemma A.8 


\subsection{Special semimartingales}

To prepare our study of exponential semimartingales, we review some properties of special semimartingales. We introduce some notation but do not state any new results here. Recall that a real-valued semimartingale is called special if it can be written as $X=X_{0}+M+V$ for some local martingale $M$ and some predictable process $V$ of finite variation, both starting at 0 . Put differently, $X$ is a special semimartingale if there exists a predictable process $V \in \mathcal{V}$ such that $X-X_{0}-$

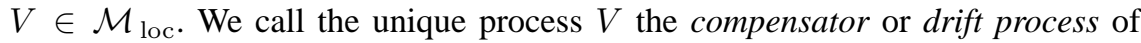
$X$ and we write $D^{X}:=V$ (for $d$ rift or Doob-Meyer decomposition). Whether a semimartingale is special or not, is an integrability property of the big jumps as the following lemma shows.

Lemma 2.8 Let $X$ be a real-valued semimartingale. Denote by $\nu^{X}$ the compensator of the measure of jumps of $X$. Then the following statements are equivalent.

\section{1. $X$ is special.}

2. $\left(|x|^{2} \wedge|x|\right) * \nu^{X} \in \mathcal{V}$

3. $1_{\{|x|>1\}}|x| * \nu^{X} \in \mathcal{V}$

Proof The equivalence $1 \Leftrightarrow 2$ is stated in JS, II.2.29. Note that predictable processes in $\mathcal{V}$ belong to $\mathcal{A}_{\text {loc }}$ (cf., JS, I.3.10). The equivalence $2 \Leftrightarrow 3$ follows from the fact that $\left(|x|^{2} \wedge 1\right) * \nu^{X} \in \mathcal{V}$ for any semimartingale $X$ (cf., JS, II.2.13).

For the following, let us slightly extend the notion of a drift process.

Definition 2.9 Let $X$ be a semimartingale in $\mathbb{R}^{d}$ and $\vartheta \in L(X)$ such that $\vartheta^{\top} \cdot X$ is a special semimartingale. Then we call its compensator the drift process of $X$ in $\vartheta$ and we write $D^{X}(\vartheta):=D^{\vartheta^{\top} \cdot X}$.

Remark If $X$ and $\vartheta^{\top} \cdot X$ in the previous definition are special, then $D^{X}(\vartheta)=$ $\vartheta^{\top} \cdot D^{X}$ (cf., Jacod 1980, Proposition 2). Here, special semimartingale and drift process in $\mathbb{R}^{d}$ are to be interpreted componentwise. Note, however, that $\vartheta^{\top} \cdot X$ may not be special even if $X$ is special (and vice versa).

A simple property of drift processes is given in the following

Lemma 2.10 Let $\vartheta \in L(X), \lambda \in L\left(\vartheta^{\top} \cdot X\right)$ be such that $(\lambda \vartheta)^{\top} \cdot X=\lambda \cdot\left(\vartheta^{\top} \cdot X\right)$ is special. Then $D^{\vartheta^{\top} \cdot X}(\lambda)=D^{X}(\lambda \vartheta)$.

Proof This follows from $\lambda \cdot\left(\vartheta^{\top} \cdot X\right)=(\lambda \vartheta)^{\top} \cdot X$ (cf., e.g., Goll and Kallsen (2000), Proposition A.1).

The drift process can be expressed explicitly in terms of semimartingale characteristics. Let $X$ be a semimartingale in $\mathbb{R}^{d}$. We assume that the characteristics $(B, C, \nu)$ of $X$ relative to some fixed truncation function $h: \mathbb{R}^{d} \rightarrow \mathbb{R}^{d}$ are given in the form

$$
B=b \cdot A, \quad C=c \cdot A, \quad \nu=A \otimes F,
$$

where $A \in \mathcal{A}_{\mathrm{loc}}^{+}$is a predictable process, $b$ is a predictable $\mathbb{R}^{d}$-valued process, $c$ is a predictable $\mathbb{R}^{d \times d}$-valued process whose values are non-negative, symmetric matrices, and $F$ is a transition kernel from $\left(\Omega \times \mathbb{R}_{+}, \mathcal{P}\right)$ into $\left(\mathbb{R}^{d}, \mathcal{B}^{d}\right)$. By JS, Proposition II.2.9 such a representation always exists. 
Lemma 2.11 Let $\vartheta \in L(X)$ such that $\vartheta^{\top} \cdot X$ is special. Then we have:

1. $D^{X}(\vartheta)=\delta(\vartheta) \cdot A$, where $\delta(\vartheta)_{t}:=\vartheta_{t}^{\top} b_{t}+\int \vartheta_{t}^{\top}(x-h(x)) F_{t}(d x)$

2. $\Delta D^{X}(\vartheta)_{t}=\int \vartheta_{t}^{\top} x \nu(\{t\} \times d x)$

Proof For real-valued $X$ and $\vartheta=1 \mathrm{cf} .$, JS, II.2.29. In the general case, observe that the characteristics of $\vartheta^{\top} \cdot X$ relative to some truncation function $h_{1}: \mathbb{R} \rightarrow \mathbb{R}$ are of the form (2.1) with $\bar{b}_{t}=\vartheta_{t}^{\top} b_{t}+\int\left(h_{1}\left(\vartheta_{t}^{\top} x\right)-\vartheta_{t}^{\top} h(x)\right) F_{t}(d x)$ and $\bar{F}_{t}(G)=$ $\int 1_{G}\left(\vartheta_{t}^{\top} x\right) F_{t}(d x)$ instead of $b_{t}$ and $F_{t}(\cdot)$ (cf., Kallsen and Shiryaev 2000, Lemma 2.5).

\subsection{Exponentially special semimartingales}

A main subject of this paper are exponentially special semimartingales in the sense of the following

Definition 2.12 Let $X$ be a real-valued semimartingale. $X$ is called exponentially special if $\exp \left(X-X_{0}\right)$ is a special semimartingale.

As in the case of special semimartingales (cf., Lemma 2.8), this property can be expressed in terms of the compensated measure of jumps $\nu^{X}$ of $X$.

Lemma 2.13 Let $X$ be a real-valued semimartingale. Denote by $\nu^{X}$ the compensator of the measure of jumps of $X$ and by $h: \mathbb{R} \rightarrow \mathbb{R}$ a truncation function. Then the following statements are equivalent.

1. $X$ is exponentially special.

2. $\left(e^{x}-1-h(x)\right) * \nu^{X} \in \mathcal{V}$

3. $1_{\{x>1\}} e^{x} * \nu^{X} \in \mathcal{V}$

Proof Set $\tilde{X}:=\mathcal{L}\left(\exp \left(X-X_{0}\right)\right)$. By Jacod (1979), (2.51), $\widetilde{X}$ is a special semimartingale if and only if $\exp \left(X-X_{0}\right)$ is a special semimartingale.

$1 \Rightarrow 3$ : By Statement 3 in Lemma 2.6 we have that $\Delta \widetilde{X}=e^{\Delta X}-1$. From Lemma 2.8 follows that $\left|e^{x}-1\right| 1_{\left\{\left|e^{x}-1\right|>1\right\}} * \nu^{X}=|x| 1_{\{|x|>1\}} * \nu^{\widetilde{X}} \in \mathcal{V}$. In particular, we have $\left(e^{x}-1\right) 1_{\{x>1\}} * \nu^{X} \in \mathcal{V}$. Since $1_{\{x>1\}} * \nu^{X} \in \mathcal{V}$, this implies $e^{x} 1_{\{x>1\}} * \nu^{X} \in \mathcal{V}$.

$3 \Rightarrow 2$ : Note that $0 \leq e^{x}-1-h(x) \leq e^{x} 1_{\{x>1\}}+M\left(1 \wedge x^{2}\right)$ for some constant $M \in \mathbb{R}_{+}$which is independent of $x \in \mathbb{R}$. Since $\left(1 \wedge x^{2}\right) * \nu^{X} \in \mathcal{V}$ holds for any semimartingale, $1_{\{x>1\}} e^{x} * \nu^{X} \in \mathcal{V}$ implies $\left(e^{x}-1-h(x)\right) * \nu^{X} \in \mathcal{V}$.

$2 \Rightarrow 1$ : Statement 1 in Lemma 2.6 implies that $\widetilde{X}=-X_{0}+\frac{1}{2}\left\langle X^{c}, X^{c}\right\rangle+\left(e^{x}-\right.$ $1-h(x)) *\left(\mu^{X}-\nu^{X}\right)+\left(e^{x}-1-h(x)\right) * \nu^{X}+\left(X-(x-h(x)) * \mu^{X}\right)$. Since all components are special semimartingales, this is true for $\widetilde{X}$ as well.

The counterpart of the compensator of a special semimartingale is defined in the following

Definition 2.14 Let $X$ be a real-valued semimartingale. A predictable process $V \in$ $\mathcal{V}$ is called exponential compensator of $X$ if $\exp \left(X-X_{0}-V\right) \in \mathcal{M}_{\text {loc }}$. 
Put differently, we decompose $\exp \left(X-X_{0}\right)=M U$ where $M \in \mathcal{M}_{\text {loc }}$ and $U=\exp (V)$ is a positive predictable process of finite variation. Well-known results on the multiplicative decomposition of semimartingales yield that such a representation exists if and only if $\exp \left(X-X_{0}\right)$ is a special semimartingale:

Lemma 2.15 A real-valued semimartingale $X$ has an exponential compensator if and only if it is exponentially special. In this case, the exponential compensator is up to indistinguishability unique.

Proof Suppose that $X$ is exponentially special. By Jacod (1979), (6.19), there exists a unique positive process $U$ of finite variation such that $U_{0}=1$ and $M:=$ $\frac{\exp \left(X-X_{0}\right)}{U} \in \mathcal{M}_{\text {loc }}$. Since $\exp \left(X-X_{0}\right)>0$ and $\frac{\exp \left(X_{-}-X_{0}\right)}{U_{-}}=M_{-}<\infty$, we have $U_{-}>0$. Therefore, $V:=\log (U) \in \mathcal{V}$.

Conversely, suppose that $V$ is an exponential compensator of $X$. Since $\exp (V)$ is of finite variation, the special semimartingale property of $X$ and the uniqueness of $V$ follows again from Jacod (1979), VI.2a and (6.19).

In the following, we want to determine exponential compensators explicitly.

\subsection{Laplace cumulant processes}

For $\mathbb{R}^{d}$-valued infinitely divisible random variables $X$, we have $E\left(e^{\vartheta^{\top} X}\right)=$ $\exp (k(\vartheta))$ for $\vartheta \in \mathbb{R}^{d}$, where $k(\vartheta)$ denotes the cumulant of $X$ in $\vartheta$ (if it exists). Below, this notion is generalized to semimartingales and leads to the so-called Laplace cumulant process. A modification of this process allows to determine exponential compensators explicitly. For a closely related complex counterpart (the Fourier cumulant process) we refer the reader to Kallsen and Shiryaev (2000).

Let $X$ be a semimartingale in $\mathbb{R}^{d}$. We assume that the characteristics $(B, C, \nu)$ of $X$ are given in the form (2.1).

\section{Definition 2.16}

1. Let $\vartheta \in L(X)$ such that $\vartheta^{\top} \cdot X$ is exponentially special. The Laplace cumulant process $\widetilde{K}^{X}(\vartheta)$ of $X$ in $\vartheta$ is defined as the compensator of the special semimartingale $\left(\vartheta^{\top} \cdot X\right)^{\sim}=\mathcal{L}\left(\exp \left(\vartheta^{\top} \cdot X\right)\right)$, i.e. $\widetilde{K}^{X}(\vartheta):=D^{\left(\vartheta^{\top} \cdot X\right)^{\sim}}$. For $\vartheta=1$ we write $\widetilde{K}^{X}:=\widetilde{K}^{X}(1)$.

2. The modified Laplace cumulant process $K^{X}(\vartheta)$ of $X$ in $\vartheta$ is the logarithmic transform of $\widetilde{K}^{X}(\vartheta)$, i.e. $K^{X}(\vartheta):=\log \left(\mathcal{E}\left(\widetilde{K}^{X}(\vartheta)\right)\right)$. For $\vartheta=1$ we write $K^{X}:=K^{X}(1)$. 


\section{Remarks}

1. From the proof of Statement 2 in Theorem 2.18 below it follows that $K^{X}(\vartheta)$ is a well-defined predictable process of finite variation.

2. Observe that the mappings $\widetilde{X} \mapsto D^{\widetilde{X}}$ and $X \mapsto K^{X}$ are dual in the sense that $\left(K^{X}\right)^{\sim}=D^{\tilde{X}}$, where $\sim$ denotes the exponential transform.

3. A theorem by Yor states that $\mathcal{E}(\tilde{X}) \mathcal{E}(\tilde{Y})=\mathcal{E}(\tilde{X}+\tilde{Y}+[\tilde{X}, \tilde{Y}])$ for any realvalued semimartingales $\widetilde{X}, \widetilde{Y}$ (cf., Jacod 1979, (6.4)). This fact can also be interpreted in terms of a duality: A simple calculation shows that the mappings $(\widetilde{X}, \widetilde{Y}) \mapsto \widetilde{X}+\widetilde{Y}+[\widetilde{X}, \widetilde{Y}]$ and $(X, Y) \mapsto X+Y$ are dual in the sense that $(X+Y)^{\sim}=\tilde{X}+\widetilde{Y}+[\tilde{X}, \tilde{Y}]$.

The following two statements summarize some properties of the cumulant processes and their explicit form in terms of the characteristics of $X$. They correspond to Lemmas 2.10 and 2.11, respectively.

Lemma 2.17 Let $\vartheta \in L(X), \lambda \in L\left(\vartheta^{\top} \cdot X\right)$ be such that $(\lambda \vartheta)^{\top} \cdot X=\lambda \cdot\left(\vartheta^{\top} \cdot X\right)$ is exponentially special. Then $\widetilde{K}^{\vartheta^{\top} \cdot X}(\lambda)=\widetilde{K}^{X}(\lambda \vartheta)$ and $K^{\vartheta^{\top} \cdot X}(\lambda)=K^{X}(\lambda \vartheta)$.

Proof This follows from $\exp \left(\lambda \cdot\left(\vartheta^{\top} \cdot X\right)\right)=\exp \left((\lambda \vartheta)^{\top} \cdot X\right)$ (cf., e.g., Goll and Kallsen 2000, Proposition A.1).

Theorem 2.18 Let $\vartheta \in L(X)$ such that $\vartheta^{\top} \cdot X$ is exponentially special. Then the following statements hold.

1. $\widetilde{K}^{X}(\vartheta)=\widetilde{\kappa}(\vartheta) \cdot A$, where $\widetilde{\kappa}(\vartheta)_{t}:=\vartheta_{t}^{\top} b_{t}+\frac{1}{2} \vartheta_{t}^{\top} c_{t} \vartheta_{t}+\int\left(e^{\vartheta_{t}^{\top} x}-1-\right.$ $\left.\vartheta_{t}^{\top} h(x)\right) F_{t}(d x)$

2. $K^{X}(\vartheta)=\widetilde{K}^{X}(\vartheta)+\sum_{s \leq .}\left(\log \left(1+\Delta \widetilde{K}^{X}(\vartheta)_{s}\right)-\Delta \widetilde{K}^{X}(\vartheta)_{s}\right)$

3. $\widetilde{K}^{X}(\vartheta)=K^{X}(\vartheta)+\sum_{s<.}\left(e^{\Delta K^{X}(\vartheta)_{s}}-1-\Delta K^{X}(\vartheta)_{s}\right)$

4. $\Delta \widetilde{K}^{X}(\vartheta)_{t}=\int\left(e^{\vartheta^{\top} x}-1\right) \nu(\{t\} \times d x)=: \widehat{W}(\vartheta)_{t}$

5. $\Delta K^{X}(\vartheta)_{t}=\log \left(1+\Delta \widetilde{K}^{X}(\vartheta)_{t}\right)=\log \left(1+\widehat{W}(\vartheta)_{t}\right)$

6. If $X$ is quasi-left-continuous, then $K^{X}(\vartheta)=\widetilde{K}^{X}(\vartheta)$.

\section{Proof}

1. By Lemma 2.6, we have $\left(\vartheta^{\top} \cdot X\right)^{\sim}=\vartheta^{\top} \cdot X+\frac{1}{2}\left\langle\vartheta^{\top} \cdot X^{c}, \vartheta^{\top} \cdot X^{c}\right\rangle+\left(e^{x}-\right.$ $1-x) * \mu^{\vartheta^{\top} \cdot X}$. From Goll and Kallsen (2000), Propositions A.2 and A.3, it follows that $\vartheta^{\top} \cdot X=\vartheta^{\top} \cdot X^{c}+\vartheta^{\top} x 1_{\Delta^{C}} *\left(\mu^{X}-\nu\right)+\vartheta^{\top} x 1_{\Delta}(x) * \mu^{X}+$ $\vartheta^{\top} \cdot \widetilde{B}$ for some $\Delta \in \mathcal{P} \otimes \mathcal{B}^{d}$ and $\widetilde{B}=B+\left(x 1_{\Delta^{c}}(x)-h(x)\right) * \nu$. Since $\left\langle\vartheta^{\top} \cdot X^{c}, \vartheta^{\top} \cdot X^{c}\right\rangle=\left(\vartheta^{\top} c \vartheta\right) \cdot A$ and $\left(e^{x}-1-x\right) * \mu^{\vartheta^{\top} \cdot X}=\left(e^{x}-1-\right.$ $x) *\left(\mu^{\vartheta^{\top} \cdot X}-\nu^{\vartheta^{\top} \cdot X}\right)+\left(e^{\vartheta^{\top} x}-1-\vartheta^{\top} x\right) * \nu$, straightforward calculations yield that $\left(\vartheta^{\top} \cdot X\right)^{\sim}$ equals $\left(\vartheta^{\top} b+\frac{1}{2} \vartheta^{\top} c \vartheta+\int\left(e^{\vartheta^{\top} x}-1-\vartheta^{\top} x 1_{\Delta}(x)-\right.\right.$ $\left.\left.\vartheta^{\top} h(x)\right) F(d x)\right) \cdot A+\vartheta^{\top} x 1_{\Delta}(x) * \mu^{X}$ up to a local martingale. Since $\left(\vartheta^{\top} \cdot X\right)^{\sim}$ is a special semimartingale, JS, I.4.23(iii) yields that $\vartheta^{\top} x 1_{\Delta}(x) * \mu^{X} \in \mathcal{A}_{\text {loc }}$ and hence $\left|\vartheta^{\top} x 1_{\Delta}(x)\right| * \mu^{X} \in \mathcal{A}_{\text {loc }}^{+}$. This in turn means $\vartheta^{\top} x 1_{\Delta}(x) * \mu^{X}=$ $\vartheta^{\top} x 1_{\Delta}(x) *\left(\mu^{X}-\nu\right)+\vartheta^{\top} x 1_{\Delta}(x) * \nu$ (cf., JS, II.1.28). Together, we have that $\left(\vartheta^{\top} \cdot X\right)^{\sim}$ equals $\left(\vartheta^{\top} b+\frac{1}{2} \vartheta^{\top} c \vartheta+\int\left(e^{\vartheta^{\top} x}-1-\vartheta^{\top} h(x)\right) F(d x)\right) \cdot A$ up to indistinguishability, which yields Statement 1 . 
2. From Statement 1 we have $\Delta \widetilde{K}^{X}(\vartheta)_{t}=\vartheta_{t}^{\top} \Delta B_{t}+\int\left(e^{\vartheta_{t}^{\top} x}-1-\vartheta_{t}^{\top} h(x)\right) \nu(\{t\}$ $\times d x)$. Since $\Delta B_{t}=\int h(x) \nu(\{t\} \times d x)\left(\right.$ cf., JS, II.2.14), this implies $\Delta \widetilde{K}^{X}(\vartheta)_{t}$ $=\int\left(e^{\vartheta^{\top} x}-1\right) \nu(\{t\} \times d x)>-1$, hence $1+\Delta \widetilde{K}^{X}(\vartheta)_{t}>0$. From JS, I.4.63 it follows that $\mathcal{E}\left(\widetilde{K}^{X}(\vartheta)\right)=\exp \left(\widetilde{K}^{X}(\vartheta)+\sum_{s \leq .}\left(\log \left(1+\Delta \widetilde{K}^{X}(\vartheta)_{s}\right)-\right.\right.$ $\left.\Delta \widetilde{K}^{X}(\vartheta)_{s}\right)$ ), which yields Statement 2 .

3. In view of $\Delta K^{X}(\vartheta)=\Delta \widetilde{K}^{X}(\vartheta)+\log \left(1+\Delta \widetilde{K}^{X}(\vartheta)\right)-\Delta \widetilde{K}^{X}(\vartheta)=\log (1+$ $\left.\Delta \widetilde{K}^{X}(\vartheta)\right)$, this follows from Statement 2 .

4. This has been shown in the proof of Statement 2 .

5. This follows from the proof of Statement 3.

6. In view of JS, II.1.19, this follows from Statements 2 and 4.

We are now ready to determine the promised explicit form of the exponential compensator in terms of Laplace cumulant processes.

Theorem 2.19 Let $\vartheta \in L(X)$ such that $\vartheta^{\top} \cdot X$ is exponentially special. Then $K^{X}(\vartheta)$ is the exponential compensator of $\vartheta^{\top} \cdot X$. More specifically,

$$
\begin{aligned}
Z & :=\exp \left(\vartheta^{\top} \cdot X-K^{X}(\vartheta)\right) \\
& =\frac{\exp \left(\vartheta^{\top} \cdot X\right)}{\mathcal{E}\left(\widetilde{K}^{X}(\vartheta)\right)} \\
& =\mathcal{E}\left(\vartheta^{\top} \cdot X^{c}+\frac{e^{\vartheta^{\top} x}-1}{1+\widehat{W}(\vartheta)} *\left(\mu^{X}-\nu\right)\right) \in \mathcal{M}_{\text {loc }},
\end{aligned}
$$

where $\widehat{W}(\vartheta)_{t}:=\int\left(e^{\vartheta^{\top} x}-1\right) \nu(\{t\} \times d x)$.

Proof Step 1: If we set $S:=\exp \left(\vartheta^{\top} \cdot X\right)$ and denote by ${ }^{p} S$ its predictable projection, then $H:=\frac{1}{S_{-}}-\frac{1}{p_{S}}$ is locally bounded by Jacod (1979), (6.19). This implies that $H \cdot S$ is a special semimartingale (cf., Jacod 1979, (2.51)). Since ${ }^{p} S_{t}=E\left(S_{t} \mid \mathcal{F}_{t-}\right)=S_{t-}+E\left(\Delta S_{t} \mid \mathcal{F}_{t-}\right)=S_{t-}\left(1+E\left(e^{\vartheta_{t}^{\top} \Delta X_{t}}-1 \mid \mathcal{F}_{t-}\right)\right)=$ $S_{t-}\left(1+\widehat{W}(\vartheta)_{t}\right)$ by JS, I.2.28 and II.1.26, it follows that $H=\frac{1}{S_{-}}\left(1-\frac{1}{1+\widehat{W}(\vartheta)}\right)=$ $\frac{1}{S_{-}} \frac{\widehat{W}(\vartheta)}{1+\widehat{W}(\vartheta)}$. If $D^{H \cdot S}$ denotes the drift process of $H \cdot S$, we have that $\Delta D^{H \cdot S}=$ $p\left(\Delta D^{H \cdot S}\right)=p(\Delta(H \cdot S))=H^{p}(\Delta S)=H\left({ }^{p} S-S_{-}\right)=\frac{(\widehat{W}(\vartheta))^{2}}{1+\widehat{W}(\vartheta)}$. In particular, we have $\left|\left(e^{\vartheta^{\top} x}-1\right) \frac{\widehat{W}(\vartheta)}{1+\widehat{W}(\vartheta)}\right| * \nu=\sum_{s \leq} \frac{\left(\widehat{W}(\vartheta)_{s}\right)^{2}}{1+\widehat{W}(\vartheta)_{s}}=\sum_{s \leq .} \Delta D_{s}^{H \cdot S} \in \mathcal{V}$. It follows that $\left(e^{\vartheta^{\top} x}-1\right) \frac{\widehat{W}(\vartheta)}{1+\widehat{W}(\vartheta)} \in G_{\text {loc }}\left(\mu^{X}\right)$ and

$$
\left(e^{\vartheta^{\top} x}-1\right) \frac{\widehat{W}(\vartheta)}{1+\widehat{W}(\vartheta)} *\left(\mu^{X}-\nu\right)=\sum_{s \leq .}\left(e^{\vartheta_{s}^{\top} \Delta X_{s}}-1-\widehat{W}(\vartheta)_{s}\right) \frac{\widehat{W}(\vartheta)_{s}}{1+\widehat{W}(\vartheta)_{s}}
$$

(cf., JS, I.3.10 and II.1.28).

Step 2: Define $Y:=\vartheta^{\top} \cdot X-K^{X}(\vartheta)$. By Statement 1 in Lemma 2.6 we have that $\mathcal{L}(\exp (Y))=\vartheta^{\top} \cdot X-K^{X}(\vartheta)+\frac{1}{2}\left\langle\vartheta^{\top} \cdot X^{c}, \vartheta^{\top} \cdot X^{c}\right\rangle+\sum_{s \leq}\left(e^{\Delta Y_{s}}-1-\right.$ $\left.\Delta Y_{s}\right)$. Since $e^{\Delta Y_{s}}-1-\Delta Y_{s}=e^{\Delta\left(\vartheta^{\top} \cdot X\right)_{s}}-1-\Delta\left(\vartheta^{\top} \cdot X\right)_{s}+\Delta K^{X}(\vartheta)_{s}+$ 
$e^{\Delta\left(\vartheta^{\top} \cdot X\right)_{s}}\left(e^{-\Delta K^{X}(\vartheta)_{s}}-1\right)$ and in view of Theorem 2.18 and Step 1, straightforward calculations yield $\mathcal{L}(\exp (Y))=\vartheta^{\top} \cdot X+\frac{1}{2}\left\langle\vartheta^{\top} \cdot X^{c}, \vartheta^{\top} \cdot X^{c}\right\rangle+\left(e^{\vartheta^{\top} x}-1-\right.$ $\left.\vartheta^{\top} x\right) * \mu^{X}-\widetilde{K}^{X}(\vartheta)-\left(e^{\vartheta^{\top} x}-1\right) \frac{\widehat{W}(\vartheta)}{1+\widehat{W}(\vartheta)} *\left(\mu^{X}-\nu\right)$. From Goll and Kallsen (2000), Propositions A.2 and A.3, it follows that $\vartheta^{\top} \cdot X=\vartheta^{\top} \cdot X^{c}+\vartheta^{\top} x 1_{\Delta^{C}} *\left(\mu^{X}-\nu\right)+$ $\vartheta^{\top} x 1_{\Delta}(x) * \mu^{X}+\vartheta^{\top} \cdot \widetilde{B}$ for some $\Delta \in \mathcal{P} \otimes \mathcal{B}^{d}$ and $\widetilde{B}=B+\left(x 1_{\Delta^{C}}(x)-h(x)\right) * \nu$. Similarly, Statement 1 in Theorem 2.18 and Proposition A.3 in Goll and Kallsen (2000) yield $\widetilde{K}^{X}(\vartheta)=\vartheta^{\top} \cdot \widetilde{B}+\frac{1}{2}\left\langle\vartheta^{\top} \cdot X^{c}, \vartheta^{\top} \cdot X^{c}\right\rangle+\left(e^{\vartheta^{\top} x}-1-\vartheta^{\top} 1_{\Delta^{C}}(x)\right) * \nu$. Together, it follows that $\mathcal{L}(\exp (Y))=\vartheta^{\top} \cdot X^{c}+\frac{e^{\vartheta^{\top} x}-1}{1+\widehat{W}(\vartheta)} *\left(\mu^{X}-\nu\right) \in \mathcal{M}_{\text {loc }}$. In view of $\exp \left(K^{X}(\vartheta)\right)=\mathcal{E}\left(\widetilde{K}^{X}(\vartheta)\right)$, the proof is complete.

If $Z$ is the density process of a probability measure $P^{\prime} \stackrel{\text { loc }}{\ll} P$, then the following result is useful for the application of Girsanov's theorem for semimartingales as in JS, III.3.24.

Lemma 2.20 Let $\vartheta$ and $Z$ be as in Theorem 2.19. Define $\beta:=\vartheta$ and the $\widetilde{\mathcal{P}}$ measurable function $Y: \Omega \times \mathbb{R}_{+} \times \mathbb{R}^{d} \rightarrow \mathbb{R}_{+}$by

$$
Y(t, x):=\frac{e^{\vartheta^{\top} x}}{1+\widehat{W}(\vartheta)_{t}} .
$$

If $M_{\mu^{X}}^{P}(\cdot \mid \widetilde{\mathcal{P}})$ is defined as in JS, III.3c and if we set $a_{t}:=\nu\left(\{t\} \times \mathbb{R}^{d}\right)$ and $\widehat{Y}_{t}:=\int Y(t, x) \nu(\{t\} \times d x)$, then we have

1. (a) $Y Z_{-}=M_{\mu^{X}}^{P}(Z \mid \widetilde{\mathcal{P}})$,

(b) $\left\langle Z^{c}, X^{i, c}\right\rangle=\left(Z_{-} c^{i \cdot \beta}\right) \cdot$ Afor $i=1, \ldots, d$,

2. $Z=\mathcal{E}\left(\beta^{\top} \cdot X^{c}+\left(Y-1+\frac{\widehat{Y}-a}{1-a} 1_{\{a<1\}}\right) *\left(\mu^{X}-\nu\right)\right)$.

\section{Proof}

1. Note that $Z=Z_{-} \exp \left(\vartheta^{\top} \Delta X-\Delta K^{X}(\vartheta)\right)$ by definition. In view of Theorem 2.18 , this equals $Z_{-} \frac{e^{\vartheta^{\top}} \Delta X}{1+\widehat{W}(\vartheta)}$. Moreover, we have $x=\Delta X_{t}(\omega)$ for $M_{\mu^{X}}^{P}$-almost all $(\omega, t, x) \in \Omega \times \mathbb{R}_{+} \times \mathbb{R}^{d}$. Together, it follows that $U Z=U Z_{-} \frac{e^{\vartheta^{\top} x}}{1+\widehat{W}(\vartheta)}=$ $U Z_{-} Y M_{\mu^{X}}^{P}$-almost everywhere for any non-negative $\widetilde{\mathcal{P}}$-measurable function $U$. This implies the first statement.

The second statement follows immediately from $Z^{c}=Z_{-} \cdot\left(\vartheta^{\top} \cdot X^{c}\right)=$ $\left(Z_{-} \beta\right)^{\top} \cdot X^{c}$.

2. Straightforward calculations yield $\widehat{Y}=\frac{a+\widehat{W}(\vartheta)}{1+\widehat{W}(\vartheta)}$ and hence $Y-1+\frac{\widehat{Y}-a}{1-a} 1_{\{a<1\}}$ $=\frac{e^{\vartheta^{\top} x}-1}{1+\widehat{W}(\vartheta)}$ for $a \neq 1$. For $a=1$ we have $Y-1+\frac{\widehat{Y}-a}{1-a} 1_{\{a<1\}}=\frac{e^{\vartheta^{\top} x}-1}{1+\widehat{W}(\vartheta)}-$ $\frac{\widehat{W}(\vartheta)}{1+\widehat{W}(\vartheta)}$. From the definition of the stochastic integral with respect to $\mu^{X}-\nu$ it follows that $\frac{\widehat{W}(\vartheta)}{1+\widehat{W}(\vartheta)} *\left(\mu^{X}-\nu\right)=0$ because the integrand does not depend on $x$. In view of Theorem 2.19 , we are done. 
Corollary 2.21 Suppose that $P^{\prime} \stackrel{\text { loc }}{\ll} P$ is a probability measure with density process $Z$ as in Theorem 2.19. Then the $P^{\prime}$-characteristics $\left(B^{\prime}, C^{\prime}, \nu^{\prime}\right)$ of $X$ are given by

$$
\begin{aligned}
B^{\prime i} & =B^{i}+c^{i \cdot} \vartheta \cdot A+h^{i}(x)\left(\frac{e^{\vartheta^{\top} x}}{1+\widehat{W}(\vartheta)}-1\right) * \nu \text { for } i=1, \ldots, d \\
C^{\prime} & =C \\
\nu^{\prime} & =\frac{e^{\vartheta^{\top} x}}{1+\widehat{W}(\vartheta)} \cdot \nu .
\end{aligned}
$$

Proof Lemma 2.20 and JS, III.3.24

\subsection{Derivatives of cumulant processes}

Theorem 2.19 shows that cumulant processes play an important role for the study of exponential martingales. We consider now its derivative with respect to $\vartheta$ since it is needed in the following sections. By Theorem 2.18, we have that $\widetilde{K}^{X}(\vartheta)=\widetilde{\kappa}(\vartheta) \cdot A$ with

$$
\widetilde{\kappa}(\vartheta)_{t}=\vartheta_{t}^{\top} b_{t}+\frac{1}{2} \vartheta_{t}^{\top} c_{t} \vartheta_{t}+\int\left(e^{\vartheta_{t}^{\top} x}-1-\vartheta_{t}^{\top} h(x)\right) F_{t}(d x) .
$$

If we may differentiate under the integral sign, then the derivative of the mapping $\mathbb{R}^{d} \rightarrow \mathbb{R}, \vartheta \mapsto \widetilde{\kappa}(\vartheta)(\omega, t)$ equals $D \widetilde{\kappa}(\vartheta)(\omega, t)=\left(D_{1} \widetilde{\kappa}(\vartheta), \ldots, D_{d} \widetilde{\kappa}(\vartheta)\right)(\omega, t)$ with

$$
D_{i} \widetilde{\kappa}(\vartheta)_{t}=b_{t}^{i}+c_{t}^{i \cdot} \vartheta_{t}+\int\left(x^{i} e^{\vartheta_{t}^{\top} x}-h^{i}(x)\right) F_{t}(d x) .
$$

Even in cases where it is not obvious whether differentiation and integration may be exchanged, the following definition makes sense.

Definition 2.22 Let $\vartheta \in L(X)$ such that $\vartheta^{\top} \cdot X$ is exponentially special and such that $\left|x^{i} e^{\vartheta^{\top} x}-h^{i}(x)\right| * \nu \in \mathcal{V}$ for $i=1, \ldots, d$. The derivative of $\widetilde{K}^{X}$ in $\vartheta$ is defined as the $\mathbb{R}^{d}$-valued process $D \widetilde{K}^{X}(\vartheta)=\left(D_{1} \widetilde{K}^{X}(\vartheta), \ldots, D_{d} \widetilde{K}^{X}(\vartheta)\right)$ where $D_{i} \widetilde{K}^{X}(\vartheta):=D_{i} \widetilde{\kappa}(\vartheta) \cdot A$ and

$$
D_{i} \widetilde{\kappa}(\vartheta)_{t}:=b_{t}^{i}+c_{t}^{i \cdot} \vartheta_{t}+\int\left(x^{i} e^{\vartheta_{t}^{\top} x}-h^{i}(x)\right) F_{t}(d x)
$$

for $i=1, \ldots, d$.

We want to define a derivative for $K^{X}(\vartheta)$ as well. If the mapping $\mathbb{R}^{d} \rightarrow \mathbb{R}$, $\vartheta \mapsto \Delta \widetilde{K}^{X}(\vartheta)(\omega, t)=\widetilde{\kappa}(\vartheta)(\omega, t) \Delta A(\omega, t)$ is differentiable, then the mapping $\mathbb{R}^{d} \rightarrow \mathbb{R}, \vartheta \mapsto \log \left(1+\Delta \widetilde{K}^{X}(\vartheta)(\omega, t)\right)$ is differentiable as well with derivative $D\left(\log \left(1+\Delta \widetilde{K}^{X}(\vartheta)_{t}\right)\right)=\frac{1}{1+\Delta \widetilde{K}^{X}(\vartheta)_{t}} D \Delta \widetilde{K}^{X}(\vartheta)_{t}$. In view of Statement 2 in Theorem 2.18, we are led to define the derivative of $K^{X}(\vartheta)$ as follows.

Definition 2.23 Let $\vartheta \in L(X)$ such that $\vartheta^{\top} \cdot X$ is exponentially special and such that $\left|x^{i} e^{\vartheta^{\top} x}-h^{i}(x)\right| * \nu \in \mathcal{V}$ for $i=1, \ldots, d$. The derivative of $K^{X}$ in $\vartheta$ is defined as the $\mathbb{R}^{d}$-valued process $D K^{X}(\vartheta)=\left(D_{1} K^{X}(\vartheta), \ldots, D_{d} K^{X}(\vartheta)\right)$ where $D_{i} K^{X}(\vartheta):=\frac{1}{1+\widehat{W}(\vartheta)} \cdot D_{i} \widetilde{K}^{X}(\vartheta)$. 
Proposition 2.24 Let $\vartheta \in L(X)$ such that $\vartheta^{\top} \cdot X$ is exponentially special and such that $\left|x^{i} e^{\vartheta^{\top} x}-h^{i}(x)\right| * \nu \in \mathcal{V}$ for $i=1, \ldots, d$. Then the derivatives of $\widetilde{K}^{X}$ and $K^{X}$ in $\vartheta$ are well-defined predictable processes whose components are of finite variation.

Proof Since $b^{i} \cdot A=B^{i}$ and $\left(c^{i} \vartheta\right) \cdot A=\left\langle X^{i, c}, \vartheta^{\top} \cdot X^{c}\right\rangle$, it follows that $D_{i} \widetilde{K}^{X}(\vartheta)$ is a well-defined predictable process in $\mathcal{V}$.

If we set $Z:=\exp \left(\vartheta^{\top} \cdot X\right)$ and denote by ${ }^{p} Z$ its predictable projection, then $\frac{1}{p Z}$ is locally bounded by Jacod (1979), (6.19). Since ${ }^{p} Z_{t}=E\left(Z_{t} \mid \mathcal{F}_{t-}\right)=$ $Z_{t-}+E\left(\Delta Z_{t} \mid \mathcal{F}_{t-}\right)=Z_{t-}\left(1+E\left(e^{\vartheta_{t}^{\top} \Delta X_{t}}-1 \mid \mathcal{F}_{t-}\right)\right)=Z_{t-}\left(1+\widehat{W}(\vartheta)_{t}\right)$ by JS, I.2.28 and II.1.26, it follows that $\frac{1}{Z_{-}}\left(\frac{1}{1+\widehat{W}(\vartheta)}\right)$ and hence also $\frac{1}{1+\widehat{W}(\vartheta)}$ is locally bounded. Therefore $\frac{1}{1+\widehat{W}(\vartheta)} \in L\left(D_{i} K^{X}(\vartheta)\right)$ for $i=1, \ldots, d$. This implies that $D_{i} K^{X}(\vartheta)$ is a well-defined predictable process in $\mathcal{V}$ as well.

Proposition 2.25 Under the conditions of Definition 2.23 we have $D_{i} K^{X}(\vartheta)=$ $D_{i} \kappa(\vartheta) \cdot A$, where

$$
D_{i} \kappa(\vartheta)_{t}:=b_{t}^{i}+c_{t}^{i \cdot} \vartheta_{t}+\int\left(\frac{x^{i} e^{\vartheta_{t}^{\top} x}}{1+\widehat{W}(\vartheta)_{t}}-h^{i}(x)\right) F_{t}(d x) .
$$

Proof By Definitions 2.23 and 2.22, we have $D_{i} K^{X}(\vartheta)=\frac{1}{1+\widehat{W}(\vartheta)} \cdot\left(B^{i}+\vartheta^{\top}\right.$. $\left.C^{\cdot i}+\left(x^{i} e^{\vartheta^{\top} x}-h^{i}(x)\right) * \nu\right)$. Note that $\frac{\widehat{W}(\vartheta)}{1+\widehat{W}(\vartheta)} \cdot B^{i}-\left(\frac{\widehat{W}(\vartheta)}{1+\widehat{W}(\vartheta)} h^{i}(x)\right) * \nu=$ $\sum_{s \leq .} 1_{\{\widehat{W}(\vartheta) \neq 0\}} \frac{\widehat{W}(\vartheta)}{1+\widehat{W}(\vartheta)}\left(\Delta B_{s}^{i}-\int h^{i}(x) \nu(\{s\} \times d x)\right)=0$ by JS, II.2.14 and the fact that $\{\widehat{W}(\vartheta) \neq 0\}$ is a predictable thin set. Moreover, $\frac{1}{1+\widehat{W}(\vartheta)} \cdot C=C$ because $C$ is continuous. Together, it follows that $D_{i} K^{X}(\vartheta)=B^{i}+\vartheta^{\top} \cdot C^{\cdot i}+$ $\left(x^{i} \frac{e^{\vartheta^{\top} x}}{1+\widehat{W}(\vartheta)}-h^{i}(x)\right) * \nu=D_{i} \kappa(\vartheta) \cdot A$.

\section{Remarks}

1. If $X$ is quasi-left-continuous, then the derivatives of $\widetilde{K}^{X}$ and $K^{X}$ coincide.

2. Let $X$ be a real-valued exponentially special semimartingale. If $\left|x e^{x}-h(x)\right| *$ $\nu \in \mathcal{V}$, then

$$
D \widetilde{K}^{X}(1)-\widetilde{K}^{X}(1)=\frac{1}{2} C+\left((x-1) e^{x}+1\right) * \nu .
$$

3. If $Z:=e^{X}$ is a real-valued local martingale, then we have $\widetilde{K}^{X}(1)=K^{X}(1)=$ 0 and $\widehat{W}(1)=0$ (cf., Theorems 2.19 and 2.18). If $\left|x e^{x}-h(x)\right| * \nu \in \mathcal{V}$, then

$$
\begin{aligned}
D K^{X}(1)=D \widetilde{K}^{X}(1) & =\frac{1}{2} C+\left((x-1) e^{x}+1\right) * \nu \\
& =\frac{1}{2} \widetilde{C}+((1+x) \log (1+x)-x) * \widetilde{\nu},
\end{aligned}
$$

where $(\widetilde{B}, \widetilde{C}, \widetilde{\nu})$ denotes the characteristics of the local martingale $\widetilde{X}$ with $Z=\mathcal{E}(X)$ (cf., Lemma 2.6). 
The following result shows how to calculate $D K^{\vartheta^{\top} \cdot X}(1)$ in terms of $D K^{X}(\cdot)$, which is useful in view of the conditions on uniform integrability in Sect. 3.

Lemma 2.26 Let $\vartheta \in L(X)$ be such that $\vartheta^{\top} \cdot X$ is exponentially special and such that $\left|x^{i} e^{\vartheta^{\top} x}-h^{i}(x)\right| * \nu \in \mathcal{V}$ for $i=1, \ldots, d$. Moreover, suppose that $\left|\vartheta^{\top} x e^{\vartheta^{\top} x}-h_{1}\left(\vartheta^{\top} x\right)\right| * \nu \in \mathcal{V}$ for some truncation function $h_{1}: \mathbb{R} \rightarrow \mathbb{R}$. Then

$$
D \widetilde{K}^{\vartheta^{\top} \cdot X}(1)=\vartheta^{\top} \cdot D \widetilde{K}^{X}(\vartheta)
$$

and

$$
D K^{\vartheta^{\top} \cdot X}(1)=\vartheta^{\top} \cdot D K^{X}(\vartheta)
$$

Proof From Definition 2.22 and Kallsen and Shiryaev (2000), Lemma 2.5 it follows that $D \widetilde{K}^{\vartheta^{\top} \cdot X}(1)=D \bar{\kappa}(1) \cdot A$ where $D \bar{\kappa}(1):=\vartheta^{\top} b+\vartheta^{\top} c \vartheta+\int\left(\vartheta^{\top} x e^{\vartheta^{\top} x}-\right.$ $\left.\vartheta^{\top} h(x)\right) F(d x)=\vartheta^{\top} D \widetilde{\kappa}(\vartheta)$. Since $|D \bar{\kappa}(1)| \cdot A \in \mathcal{V}$, we have that $\vartheta \in L\left(D \widetilde{K}^{X}(\vartheta)\right)$ and $D \widetilde{K}^{\vartheta^{\top} \cdot X}(1)=\vartheta^{\top} \cdot D \widetilde{K}^{X}(\vartheta)$. In view of $D K^{\vartheta^{\top} \cdot X}(1)=$ $\frac{1}{1+\widetilde{W}(\vartheta)} D \widetilde{K}^{\vartheta^{\top} \cdot X}(1)$, the second statement follows as well.

\section{Uniform integrability of exponential local martingales}

In this section, we consider positive local martingales of the form $\mathcal{E}(\widetilde{X})$ or equivalently $\exp (X)$, where $X$ denotes the logarithmic transform of $\widetilde{X}$. They play an important role in statistics and finance because they appear as natural candidates for density processes. Since $\mathcal{E}(\widetilde{X})$ is a supermartingale (cf., e.g., Jacod 1979, (5.17)), it converges to some random variable $\mathcal{E}(\widetilde{X})_{\infty}$ with $E\left(\mathcal{E}(\widetilde{X})_{\infty}\right) \leq 1$. To define a probability measure $P^{\prime} \sim P$ via its density $\frac{d P^{\prime}}{d P}:=\mathcal{E}(\widetilde{X})_{\infty}$ is only possible if $\mathcal{E}(\widetilde{X})_{\infty}=1$, or equivalently, if $\mathcal{E}(\widetilde{X})$ is a uniformly integrable martingale. This explains why the question of uniform integrability has received so much attention (cf., the references in the introduction).

Roughly speaking, one may distinguish two kinds of sufficient conditions for uniform integrability. Predictable criteria (as e.g., Novikov's condition) depend only on the characteristics of $\widetilde{X}$, whereas optional conditions (as e.g., Kazamaki's condition) involve $\widetilde{X}$ directly and not only its characteristics. Our conditions below will be formulated in terms of $X$ while the literature focuses mainly on $\widetilde{X}$. In the predictable case or for continuous processes, the two viewpoints lead essentially to the same results. For processes with jumps, however, we obtain new kinds of optional criteria. Note that all proofs are relegated to the end in this section.

Our setting is as follows. Let $X$ be a $\mathbb{R}^{d}$-valued semimartingale and let $\vartheta \in$ $L(X)$ be such that $\vartheta^{\top} \cdot X$ is exponentially special. We want to obtain sufficient conditions for $\exp \left(\vartheta^{\top} \cdot X-K^{X}(\vartheta)\right) \in \mathcal{M}_{\text {loc }}$ to be a uniformly integrable martingale. Since this process equals $\exp \left(Y-K^{Y}(1)\right)$ for $Y:=\vartheta^{\top} \cdot X$, it suffices to consider the case that $X$ is $\mathbb{R}$-valued and $\vartheta=1$. The reason to consider arbitrary $\vartheta$ in the first place is that density processes of the above type appear frequently in applications (cf., Sect. 4). 
From now on let $X$ be a real-valued semimartingale that is exponentially special and whose characteristics are of the form (2.1). Set $K(\cdot):=K^{X}(\cdot)$ and

$$
Z:=\exp (X-K(1))
$$

We start by defining a number of prospective sufficient conditions for uniform integrability of $Z$.

Definition 3.1 Let $a \in[0,1], \delta \in(0,1)$. We define the following integrability conditions:

$I(a, 1+\delta)^{1+\varepsilon}:$ We say that Condition $I(a, 1+\delta)^{1+\varepsilon}$ holds if $(1+\delta) X$ is exponentially special and if there exists some $\varepsilon>0$ such that

$$
\begin{aligned}
& \sup \left\{E \left(\operatorname { e x p } \left(( 1 + \varepsilon ) \left(a\left((1+\delta) X_{T}-K(1+\delta)_{T}\right)\right.\right.\right.\right. \\
& \left.\left.\left.\left.+\frac{1-a}{\delta}\left(K(1+\delta)_{T}-(1+\delta) K(1)_{T}\right)\right)\right)\right): T \text { finite stopping time }\right\} \\
& <\infty
\end{aligned}
$$

$I(a, 1)^{1+\varepsilon}$ : We say that Condition $I(a, 1)^{1+\varepsilon}$ holds if $\left|x e^{x}-h(x)\right| * \nu \in \mathcal{V}$ and if there exists some $\varepsilon>0$ such that

$$
\begin{gathered}
\sup \left\{E\left(\exp \left((1+\varepsilon)\left(a X_{T}+(1-a) D K(1)_{T}-K(1)_{T}\right)\right)\right):\right. \\
T \text { finite stopping time }\}<\infty .
\end{gathered}
$$

$I(a, 1)$ : We say that Condition $I(a, 1)$ holds if $\left|x e^{x}-h(x)\right| * \nu \in \mathcal{V}$ and

$$
\begin{gathered}
\sup \left\{E\left(\exp \left(a X_{T}+(1-a) D K(1)_{T}-K(1)_{T}\right)\right):\right. \\
T \text { finite stopping time }\}<\infty .
\end{gathered}
$$

$I(0,1+\delta)$ : We say that Condition $I(0,1+\delta)$ holds if $(1+\delta) X$ is exponentially special and

$$
\sup _{t \in \mathbb{R}_{+}} E\left(\exp \left(\frac{1}{\delta}\left(K(1+\delta)_{t}-(1+\delta) K(1)_{t}\right)\right)\right)<\infty .
$$

$I(0,1)$ : We say that Condition $I(0,1)$ holds if $\left|x e^{x}-h(x)\right| * \nu \in \mathcal{V}$ and

$$
\sup _{t \in \mathbb{R}_{+}} E\left(\exp \left(D K(1)_{t}-K(1)_{t}\right)\right)<\infty .
$$


Table 1. Relationships

\begin{tabular}{|c|c|c|c|c|c|}
\hline 0 & $I(0,1)^{1+\varepsilon}$ & $\stackrel{3.2}{\Rightarrow}$ & & 32 & $I(0,1)$ \\
\hline & & & $I(0,1-)$ & & \\
\hline$\wedge l$ & $\Downarrow 3.4$ & & $\Downarrow 3.2$ & & $\Downarrow 3.4$ \\
\hline$a$ & $I(a, 1)^{1+\varepsilon}$ & $\Rightarrow$ & & & $I(a, 1)$ \\
\hline$\wedge 1$ & $\Downarrow 3.4$ & & & & $\Downarrow 3.4$ \\
\hline$\widetilde{a}$ & $I(\widetilde{a}, 1)^{1+\varepsilon}$ & $\Rightarrow$ & & & $I(\widetilde{a}, 1)$ \\
\hline$\wedge$ & & $\stackrel{3}{i}^{\sigma}$ & & $(3 \hat{K})$ & $\Downarrow 3.4$ \\
\hline 1 & $I(1,1)^{1+\varepsilon}$ & $\stackrel{3.3}{\Rightarrow}$ & $Z \in \mathcal{M}$ & $\stackrel{3.3}{\Rightarrow}$ & $I(1,1)$ \\
\hline
\end{tabular}

$I(0,1-)$ : We say that Condition $I(0,1-)$ holds if

$$
\lim _{\delta \downarrow 0} \sup _{t \in \mathbb{R}_{+}} \delta \log \left(E\left(\exp \left(\frac{1}{\delta}\left((1-\delta) K(1)_{t}-K(1-\delta)_{t}\right)\right)\right)\right)=0 .
$$

\section{Remarks}

1. Note that $I(0,1)$ is defined twice. But since $D K(1)-K(1)$ is an increasing process, the two definitions coincide (cf., Proposition 3.15 for $\delta=1$ ).

2. The conditions $I(a, 1+\delta)^{1+\varepsilon}$ and $I(0,1+\delta)$ are rather of auxiliary nature than of interest on their own.

For $a=0$ the above criteria depend only on the cumulant process and hence the characteristics of $X$. Therefore, they are predictable conditions which makes them handy for applications.

Theorem 3.2 We have $I(0,1)^{1+\varepsilon} \Rightarrow I(0,1) \Rightarrow I(0,1-) \Rightarrow Z \in \mathcal{M}$, where the last statement means that $Z$ is a uniformly integrable martingale.

For continuous local martingales, Condition $I(0,1)^{1+\varepsilon}$ goes back to Liptser and Shiryaev (1972). Novikov $(1972,1975)$ and Lépingle and Mémin (1978b) showed that $I(0,1)$ suffices to conclude that $Z$ is a uniformly integrable martingale. The slight generalization to $I(0,1-)$ has been shown in Yan $(1982 \mathrm{~b})$ for continuous processes. The general version of Condition $I(0,1-)$ is, to the best of our knowledge, new.

In the case $a \neq 0$ we obtain optional criteria which are usually weaker than the predictable conditions above. Therefore they may be helpful in cases where the latter do not hold. In order to understand the criteria let us start with the essentially trivial case $a=1$, where the conditions are close to the desired uniform integrability. 
Proposition 3.3 We have $I(1,1)^{1+\varepsilon} \Rightarrow Z \in \mathcal{M} \Rightarrow I(1,1)$.

Now observe that for $0<a<1$ the conditions $I(a, 1)\left(\right.$ resp. $I(a, 1)^{1+\varepsilon}$ ) are some kind of convex combination of $I(1,1)$ and $I(0,1)$ (resp. $I(1,1)^{1+\varepsilon}$ and $I(0,1)^{1+\varepsilon}$ ). The following lemma shows that the more $a$ is increased, the weaker the conditions get.

Lemma 3.4 Let $a, \tilde{a} \in[0,1]$ with $a<\widetilde{a}$.

1. If Condition $I(a, 1)^{1+\varepsilon}$ holds and $\widetilde{a}<1$, then Condition $I(\widetilde{a}, 1)^{1+\varepsilon}$ holds as well.

2. If Condition $I(a, 1)$ holds, then Condition $I(\widetilde{a}, 1)$ holds as well.

One may wonder whether Statement 1 in the previous lemma holds also for $\widetilde{a}=1$. This is not the case as the following example shows.

Example 3.5 Let $f: \mathbb{R} \rightarrow \mathbb{R}_{+}$be some continuous function with $\int f(x) d x<\infty$, $\int\left|x e^{x}\right| f(x) d x<\infty$, and $\int e^{(1+\varepsilon) x} f(x) d x=\infty$ for any $\varepsilon>0$. Moreover, let $L$ be a real-valued Lévy process with characteristic triplet $(b, 0, F)$ where $F$ has Lebesgue density $f$ and $b:=-\int\left(e^{x}-1-h(x)\right) F(d x)$. Since $K^{L}(1)=$ $\widetilde{K}^{L}(1)=0$, it follows that $e^{L}$ is a local martingale and hence a martingale (cf., e.g., Kallsen 2000, Lemma 4.4). Therefore, $\sup \left\{E\left(\exp \left(X_{T}\right)\right): T\right.$ finite stopping time $\}<\infty$ for $X:=\left(L_{t \wedge 1}\right)_{t \in \mathbb{R}_{+}}$. Note that $K^{X}(1)=0$ and $D K^{X}(1)_{t}=$ $(1 \wedge t) \int\left((x-1) e^{x}+1\right) f(x) d x$ is bounded by some constant. By choosing $1+\varepsilon<\frac{1}{a}$, it follows that Condition $I(a, 1)^{1+\varepsilon}$ holds for $X$ and any $a \in(0,1)$. However, Theorem 2 in Wolfe $(1971)$ yields that $E\left(e^{(1+\varepsilon) L_{1}}\right)=\infty$ for any $\varepsilon>0$. Hence, Condition $I(1,1)^{1+\varepsilon}$ does not hold.

Note that Condition $I(a, 1)^{1+\varepsilon}$ obviously implies $I(a, 1)$. The reason to introduce $I(a, 1)^{1+\varepsilon}$ as well is that Condition $I(a, 1)$ is only sufficient if $X$ does not have too many large negative jumps.

Theorem 3.6 Let $a \in[0,1]$. If Condition $I(a, 1)^{1+\varepsilon}$ holds, then $Z \in \mathcal{M}$.

Theorem 3.7 Suppose that $X$ is quasi-left continuous and that there exist some $m, M \in \mathbb{R}$ with $\nu\left(\mathbb{R}_{+} \times(-\infty, m]\right)<M$ P-almost surely. Let $a \in[0,1)$. If Condition $I(a, 1)$ holds, then $Z \in \mathcal{M}$.

At first, the condition on the large negative jumps im Theorem 3.7 looks quite unnatural. However, it cannot be dropped in general. In the following example, $I(a, 1)$ holds for any $a \in[0,1]$, but $Z$ is not a uniformly integrable martingale.

Example 3.8 Let $N$ be a standard Poisson process in the sense of JS, I.3.26 and denote by $T$ its first jump time. Define $X:=-\int_{0}^{\cdot} t d N_{t}^{T}$. Obviously, $X$ is exponentially special and $K(a)_{t}=\left(e^{a x}-1\right) * \nu_{t}=-\int_{0}^{t \wedge T}\left(1-e^{-a s}\right) d s$ for any $a \in(0,1]$. Moreover, we have $D K(1)_{t}-K(1)_{t}=\left((x-1) e^{x}+1\right) * \nu_{t}=$ $\int_{0}^{t \wedge T}\left(1-(1+s) e^{-s}\right) d s$. Note that $\left(1-(1+s) e^{-s}\right)-\left(1-e^{-a s}\right) \leq e^{-a s}$. Straightforward calculations yield $D K(1)-K(1) \leq \frac{1}{a}-K(a)$ and $-K(1) \leq \frac{1}{a}-K(a)$. 
Since $\exp (a X-K(a))$ is a supermartingale, we have $E\left(\exp \left(a X_{T}-K(a)_{T}\right)\right) \leq 1$ for any finite stopping time $T$ (cf., JS, I.1.39). This implies that

$$
\begin{aligned}
& E\left(\exp \left(a X_{T}+(1-a) D K(1)_{T}-K(1)_{T}\right)\right) \\
& =E\left(\exp \left(a X_{T}-a K(1)_{T}+(1-a)\left(D K(1)_{T}-K(1)_{T}\right)\right)\right) \\
& \leq e^{\frac{1}{a}} E\left(\exp \left(a X_{T}-K(a)_{T}\right)\right) \leq e^{\frac{1}{a}}
\end{aligned}
$$

for any finite stopping time $T$. Therefore, Condition $I(a, 1)$ holds for any $a \in(0,1]$.

On the other hand, we will prove that $Z:=\exp (X-K(1))$ is not a uniformly integrable martingale. It suffices to show that $Z_{\infty}<1 P$-almost surely, where $Z_{\infty}$ denotes the limit of the positive supermartingale $Z$. On $[0, T)$ we have $N_{t}=0$ and hence $X_{t}-K(1)_{t}=t-\left(1-e^{-t}\right)$. From $\Delta X_{T}=-T$ it follows that $X_{T}-K(1)_{T}=X_{T-}-K(1)_{T-}+\Delta X_{T}=-\left(1-e^{-T}\right)<0$ on $\{0<T<\infty\}$. Since $X-K(1)=(X-\widetilde{K}(1))^{T}$, we have $Z_{\infty}<e^{0}=1$ on $\{0<T<\infty\}$. Therefore, it suffices to prove that $0<T<\infty P$-almost surely. But this is evident because $T$ is the first jump time of a standard Poisson process.

Let us relate Theorems 3.6 and 3.7 to the literature. Kazamaki's criterion for continuous local martingales is equivalent to Condition $I\left(\frac{1}{2}, 1\right)$ (cf., Kazamaki 1979). Similarly, the subsequent generalization by Kazamaki and Sekiguchi (1982) corresponds to $I(a, 1)$ in the continuous case. Note that Conditions $I(a, 1), I(a, 1)^{1+\varepsilon}$ for $a \neq 0$ depend on $X$, whereas similar criterions in the literature involve the local martingale $M:=(X-K(1))^{\sim}$ which satisfies $Z=\mathcal{E}(M)$ (cf., e.g., Lépingle and Mémin 1978b,a; Okada 1982; Yan 1982a). Since $X$ and $M$ coincide only in the continuous case, one cannot generally compare these two kinds of conditions. We feel that the conditions derived from $X$ instead of $M$ are the more natural ones.

Counterexamples in Lépingle and Mémin (1978b), VI.2 and Jacod (1979), Exercices 8.12-8.14 show that Condition $I(0,1)$ is "optimal" in the following sense. For any $\varepsilon \in(0,1)$, they present continuous and discontinuous processes $X$ such that $Z=\exp (X-K(1))$ is not a uniformly integrable martingale but

$$
\sup _{t \in \mathbb{R}_{+}} E\left(\exp \left((1-\varepsilon)\left(D K(1)_{t}-K(1)_{t}\right)\right)\right)<\infty
$$

holds. This implies that Condition $I(0,1)$ cannot be improved by a factor $1-\varepsilon$. From (3.1) and Proposition 3.11 it follows that

$$
\begin{gathered}
\sup \left\{E\left(\exp \left((1-\varepsilon)\left(a X_{T}+(1-a) D K(1)_{T}-K(1)_{T}\right)\right)\right):\right. \\
T \text { finite stopping time }\}<\infty
\end{gathered}
$$

for any $a \in[0,1]$. Therefore Condition $I(a, 1)$ cannot be improved by a factor $1-\varepsilon$ either. 
In the following example, Kazamaki-type criterions are derived for a particular class of pure jump processes.

Example 3.9 Let $\widetilde{X}$ be a purely discontinuous local martingale with jumps of fixed size $\widetilde{x} \in(-1, \infty) \backslash\{0\}$. Set $Z:=\mathcal{E}(\widetilde{X})$. We want to determine a factor $\alpha>0$ such that the condition

$$
\sup \left\{E\left(\exp \left(\alpha \widetilde{X}_{T}\right)\right): T \text { finite stopping time }\right\}<\infty
$$

suffices to ensure that $Z$ is a uniformly integrable martingale. (Note that for continuous local martingales, the "right" factor is $\alpha=\frac{1}{2}$ by Kazamaki's criterion.)

Firstly, we write the compensator of the measure of jumps of $\widetilde{X}$ as $\nu^{\widetilde{X}}=A \otimes \varepsilon \widetilde{x}$ with $A \in \mathcal{A}_{\text {loc }}$. Note that $\widetilde{X}$ is quasi-left-continuous, because $\int x \nu^{\widetilde{X}}(\{T\} \times d x)=$ $E\left(\Delta \widetilde{X}_{T} \mid \mathcal{F}_{T-}\right)=0$ implies $\nu^{\widetilde{X}}(\{(T, \widetilde{x})\})=0$ for any predictable stopping time $T$ (cf., JS, II.1.26, I.2.27, II.1.17, II.1.19). If $X$ denotes the logarithmic transform of $\widetilde{X}$, then $\mathcal{E}(\widetilde{X})=\exp (X)$ and $K^{X}(1)=0$ by Theorem 2.19. The application of Remark 3 following Proposition 2.25 yields $D K^{X}(1)=((1+\widetilde{x}) \log (1+$ $\widetilde{x})-\widetilde{x}) A$. Starting e.g. from Lemma 2.6, straightforward calculations yields that $X=\frac{\log (1+\widetilde{x})}{\widetilde{x}} \widetilde{X}+(\log (1+\widetilde{x})-\widetilde{x}) A$. By Theorem 3.7, a sufficient criterion for $Z \in \mathcal{M}$ is $\sup \left\{E\left(\exp \left(a X_{T}+(1-a) D K^{X}(1)\right)\right): T\right.$ finite stopping time $\}<\infty$. If we choose $a:=\frac{(1+\widetilde{x}) \log (1+\widetilde{x})-\widetilde{x}}{\widetilde{x} \log (1+\widetilde{x})}$, then $a X_{T}+(1-a) D \widetilde{K}^{X}(1)$ is a multiple of $\widetilde{X}$. It follows that a sufficiently large factor in Condition (3.2) is

$$
\alpha:=\frac{(1+\widetilde{x}) \log (1+\widetilde{x})-\widetilde{x}}{\widetilde{x}^{2}} .
$$

Note that $\alpha \approx \frac{1}{2}$ if the jump size is close to 0 , which corresponds approximately to Kazamaki's criterion for continuous local martingales. In Yan (1982a), (7), the factor $\max \left(1, \frac{1}{2+\widetilde{x}}\right)$ is proposed, which is strictly larger than $\alpha$. The criterion in Okada (1982) leads to the factor $\alpha$ above, but he requires uniform integrability of the set $\left\{\exp \left(\alpha \widetilde{X}_{T}\right): T\right.$ finite stopping time $\}$, which is slightly stronger.

Let us summarize the sufficient conditions of this section in the following

Corollary 3.10 Let $a \in[0,1)$. For $Z$ to be a uniformly integrable martingale, any of the following conditions suffices.

1. $I(0,1)$

2. $I(0,1-)$

3. $I(a, 1)^{1+\varepsilon}$

4. $I(a, 1)$ if $X$ is quasi-left continuous and if there exist some $m, M \in \mathbb{R}$ with $\nu\left(\mathbb{R}_{+} \times(-\infty, m]\right)<M P$-almost surely

Table 1 provides an overview about the relationships between the conditions in this paper. 


\subsection{Proofs}

We start with some auxiliary results.

Proposition 3.11 Let $\left(U_{i}\right)_{i \in I},\left(V_{i}\right)_{i \in I}$ be families of random variables, moreover $a, \widetilde{a} \in[0,1]$ with $a<\widetilde{a}$.

1. Let $\widetilde{a}<1$ and $\varepsilon \in(0,1]$. If $\sup _{i \in I} E\left(\exp \left(U_{i}\right)\right)<\infty$ and $\sup _{i \in I} E(\exp ((1+$ $\left.\left.\varepsilon)\left(a U_{i}+(1-a) V_{i}\right)\right)\right)<\infty$, then there exists some $\widetilde{\varepsilon} \in(0,1]$ such that $\sup _{i \in I} E\left(\exp \left((1+\widetilde{\varepsilon})\left(\widetilde{a} U_{i}+(1-\widetilde{a}) V_{i}\right)\right)\right)<\infty$.

2. If $\sup _{i \in I} E\left(\exp \left(U_{i}\right)\right)<\infty$ and $\sup _{i \in I} E\left(\exp \left(a U_{i}+(1-a) V_{i}\right)\right)<\infty$, then $\sup _{i \in I} E\left(\exp \left(\left(\widetilde{a} U_{i}+(1-\widetilde{a}) V_{i}\right)\right)<\infty\right.$.

\section{Proof}

1. W.1.o.g. choose $\varepsilon$ so small that $(1+\varepsilon) \widetilde{a}<1$. Moreover, let $\widetilde{\varepsilon} \in(0, \varepsilon)$ such that $\frac{(1-\widetilde{a})(1+\widetilde{\varepsilon})}{1-(1+\widetilde{\varepsilon}) \widetilde{a}}<1+\varepsilon$. We have $\exp \left((1+\widetilde{\varepsilon})\left(\widetilde{a} U_{i}+(1-\widetilde{a}) V_{i}\right)\right)=\exp \left(\frac{\widetilde{a}-a}{1-a}(1+\right.$ $\left.\widetilde{\varepsilon}) U_{i}\right) \exp \left(\frac{1-\widetilde{a}}{1-a}(1+\widetilde{\varepsilon})\left(a U_{i}+(1-a) V_{i}\right)\right)$. If we set $\alpha:=\frac{\widetilde{a}-a \widetilde{a}}{\widetilde{a}-a}$, then $\alpha>1$ and $\frac{1-\widetilde{a}}{1-a}=1-\widetilde{a} \alpha^{-1}$. From Hölder's inequality with $\frac{1}{p}:=\frac{(1+\widetilde{\varepsilon}) \widetilde{a}}{\alpha}$ and $\frac{1}{q}:=$ $1-\frac{1}{p}:=\frac{\alpha-(1+\widetilde{\varepsilon}) \widetilde{a}}{\alpha}$ it follows that

$$
\begin{aligned}
& E\left(\exp \left((1+\widetilde{\varepsilon})\left(\widetilde{a} U_{i}+(1-\widetilde{a}) V_{i}\right)\right)\right. \\
\leq & E\left(\exp \left(U_{i}\right)\right)^{\frac{1}{p}} E\left(\exp \left(\frac{q(1+\widetilde{\varepsilon})(1-\widetilde{a})}{1-a}\left(a U_{i}+(1-a) V_{i}\right)\right)\right)^{\frac{1}{q}}
\end{aligned}
$$

Easy calculations yield that $\frac{q(1+\widetilde{\varepsilon})(1-\widetilde{a})}{1-a}=\frac{(\alpha-\widetilde{a})(1+\widetilde{\varepsilon})}{\alpha-(1+\widetilde{\varepsilon}) \widetilde{a}} \leq 1+\varepsilon$. In view of the assumptions, this implies that the right-hand side of inequality (3.3) is bounded uniformly in $i \in I$. This proves the first claim.

2. This is shown as Statement 1 if we replace $\varepsilon, \widetilde{\varepsilon}$ with 0 .

Proposition 3.12 Let $U$ be a real-valued random variable with $E\left(e^{U}\right)=1$ and $E\left(|U| e^{U}\right)<\infty$. If $\vartheta \in[0,1)$, then $\frac{1}{1-\vartheta} \log \left(E\left(e^{\vartheta U}\right)\right) \geq-E\left(U e^{U}\right)$.

Proof Step 1: We will show that the mapping $[0,1] \rightarrow \mathbb{R}, \vartheta \mapsto E\left(e^{\vartheta U}\right)$ is differentiable in 1 with derivative $E\left(U e^{U}\right)$ : For any sequence $\left(\vartheta_{n}\right)_{n \in \mathbb{N}}$ with $\vartheta_{n} \uparrow 1$ we have $\frac{e^{\vartheta_{n} U}-e^{U}}{\vartheta_{n}-1} \rightarrow U e^{U}$ for $n \rightarrow \infty$. By convexity of the mapping $\vartheta \mapsto e^{\vartheta U}$, we have $\left|\frac{e^{\vartheta_{n}}-e^{U}}{\vartheta_{n}-1}\right| \leq U e^{U}$ for $U \geq 0$ and $\left|\frac{e^{\vartheta_{n} U}-e^{U}}{\vartheta_{n}-1}\right| \leq\left|\frac{e^{0}-e^{U}}{0-1}\right| \leq 1$ for $U<0$. Hence dominated convergence yields the claim.

Step 2: Step 1 and the chain rule yield that the mapping $f:[0,1] \rightarrow \mathbb{R}, \vartheta \mapsto$ $\log \left(E\left(e^{\vartheta U}\right)\right)$ is differentiable in 1 with derivative $\frac{E\left(U e^{U}\right)}{E\left(e^{U}\right)}=E\left(U e^{U}\right)$. For $\lambda \in$ $[0,1], \vartheta, \widetilde{\vartheta} \in(0,1]$ we have $\lambda f(\vartheta)+(1-\lambda) f(\widetilde{\vartheta})=\log \left(\left(E\left(e^{\vartheta U}\right)\right)^{\lambda}\left(E\left(e^{\widetilde{\vartheta} U}\right)\right)^{1-\lambda}\right)$. By Hölder's inequality with $\frac{1}{p}:=\lambda, \frac{1}{q}:=1-\frac{1}{p}=1-\lambda$, this dominates $\log \left(E\left(\left(e^{\vartheta U}\right)^{\lambda}\left(e^{\widetilde{\vartheta} U}\right)^{1-\lambda}\right)\right)=f(\lambda \vartheta+(1-\lambda) \widetilde{\vartheta})$. Therefore $f$ is convex. Together, we obtain that $\log \left(E\left(e^{\vartheta U}\right)\right) \geq \log \left(E\left(e^{U}\right)\right)+(\vartheta-1) E\left(U e^{U}\right)=(\vartheta-1) E\left(U e^{U}\right)$ for any $\vartheta \in[0,1]$. 
Proposition 3.13 For any predictable process $V \in \mathcal{V}$ the following holds.

1. $K^{X+V}(\vartheta)=K^{X}(\vartheta)+\vartheta V$ for any $\vartheta \in \mathbb{R}$.

2. $\frac{1}{\delta}\left((1-\delta) K^{X+V}(1)-K^{X+V}(1-\delta)\right)=\frac{1}{\delta}((1-\delta) K(1)-K(1-\delta))$ for any $\delta \in(0,1)$.

3. If $(1+\delta) X$ is exponentially special, then $\frac{1}{\delta}\left(K^{X+V}(1+\delta)-(1+\delta) K^{X+V}(1)\right)$ $=\frac{1}{\delta}\left(K^{X}(1+\delta)-(1+\delta) K^{X}(1)\right)$.

4. If $\left|x e^{x}-h(x)\right| * \nu \in \mathcal{V}$, then $\left|x e^{x}-h(x)\right| * \nu^{X+V} \in \mathcal{V}$ and $D K^{X+V}(1)-$ $K^{X+V}(1)=D K(1)-K(1)$.

Proof Lemma 2.15 and Theorem 2.19 yield the first assertion, which in turn implies Statements 2 and 3. For the last assertion assume w.l.o.g. $V=-K(1)$ and denote by $(\bar{B}, \bar{C}, \bar{\nu})$ the characteristics of $X+V$. Obviously, we have $\bar{C}=C$. Since $1_{G}(\Delta(X+V))=1_{G}(\Delta X+\Delta V) 1_{\{\Delta X \neq 0\}}+1_{G}(\Delta V)\left(1-1_{\{\Delta X \neq 0\}}\right)$ for any $G \in \mathcal{B}$ with $0 \notin G$, we have $1_{G}(x) * \bar{\nu}=1_{G}(x+\Delta V) * \nu_{t}+\sum_{s \leq .} 1_{G}(\Delta V)\left(1-a_{s}\right)$, where $a_{s}:=\nu(\{s\} \times \mathbb{R})$. It follows that $\frac{1}{2} \bar{C}+\left((x-1) e^{x}+1\right) * \bar{\nu}=\frac{1}{2} C+((x-$ $\left.\Delta K(1)-1) e^{x-\Delta K(1)}+1\right) * \nu+\sum_{s \leq \cdot}\left(1-\left(\Delta K(1)_{s}-1\right) e^{-\Delta K(1)_{s}}\right)\left(1-a_{s}\right)$.

A straightforward calculation yields that this expression equals $\frac{1}{2} C+\left(\frac{x e^{x}}{1+\widehat{W}(1)}\right.$ $\left.-e^{x}+1\right) * \nu+\sum_{s \leq .}\left(\widehat{W}(1)_{s}-\log \left(1+\widehat{W}(1)_{s}\right)\right)$. In view of $D K(1)=B+C+$ $\left(\frac{x e^{x}}{1+\widehat{W}(1)}-h(x)\right) * \nu$ and $K(1)=B+\frac{1}{2} C+\left(e^{x}-1-h(x)\right) * \nu+\sum_{s \leq .}(\log (1+$ $\left.\left.\widehat{W}(1)_{s}\right)-\widehat{W}(1)_{s}\right)$ (cf., Proposition 2.25 and Theorem 2.18), it follows that $\frac{1}{2} \bar{C}+$ $\left((x-1) e^{x}+1\right) * \bar{\nu}=D K(1)-K(1) \in \mathcal{V}$. In particular, $\left((x-1) e^{x}+1\right) * \bar{\nu} \in \mathcal{V}$. Since $\left|e^{x}-1-h(x)\right| * \bar{\nu} \in \mathcal{V}$, this implies that $\left|x e^{x}-h(x)\right| * \bar{\nu}=\mid(x-1) e^{x}+$ $1+e^{x}-1-h(x) \mid * \bar{\nu} \in \mathcal{V}$. But by Remark 3 following Proposition 2.25, we have $D K^{X+V}(1)-K^{X+V}(1)=\frac{1}{2} \bar{C}+\left((x-1) e^{x}+1\right) * \bar{\nu}$, which completes the proof of Statement 4.

Proposition 3.14 Let $X$ be quasi-left-continuous and $\left|x e^{x}-h(x)\right| * \nu \in \mathcal{V}$. Moreover, suppose that there exist some $m, M \in \mathbb{R}$ with $\nu\left(\mathbb{R}_{+} \times(-\infty, m]\right)<M P$ almost surely. For any $\delta \in(0,1)$ there exists some real number $\alpha \in(0, \infty)$ such that

$$
\frac{1+\alpha}{\delta}((1-\delta) K(1)-K(1-\delta)) \leq \alpha M+(D K(1)-K(1))
$$

Proof W.1.o.g. $m \leq 0$. For $\alpha \in \mathbb{R}_{+}$set $g_{\alpha}: \mathbb{R} \rightarrow \mathbb{R}, x \mapsto e^{x}(x-1)+1-$ $(1+\alpha)\left(e^{x}\left(\frac{1-e^{-\delta x}}{\delta}-1\right)+1\right)$. Note that $g_{\alpha}(0)=0$. Moreover $g_{\alpha}^{\prime}(x) \geq e^{x}(x-$ $(1+\alpha)(1-\delta) x) \geq 0$ for $x \geq 0$ if $\alpha$ is so small that $(1+\alpha)(1-\delta) \leq 1$. Straightforward calculations yield $g_{\alpha}(x) \geq e^{x}\left(\frac{e^{-\delta x}-1+\delta x}{\delta}-\alpha\left(e^{-x}-1+x\right)\right)$ for $x \leq 0$. This implies that $g_{0}(x) \geq 0$ for any $x \in \mathbb{R}$. Since $e^{-x}-1+x$ is bounded on $[m, 0]$ and $\lim _{x \rightarrow 0}\left(\frac{e^{-\delta x}-1-\delta x}{\delta}\right) /\left(e^{-x}-1+x\right)=\delta>0$, we have $g_{\alpha}(x) \geq 0$ for $x \in[m, \infty)$ if $\alpha>0$ is chosen small enough. Now let $\alpha>0$ be so small that $(1+\alpha)(1-\delta) \leq 1$ and $g_{\alpha}(x) \geq 0$ for $x \in[m, \infty)$. Note that 
$g_{\alpha}(x)=g_{0}(x)-\alpha\left(e^{x}\left(\frac{1-e^{-\delta x}}{\delta}-1\right)+1\right) \geq-\alpha\left(e^{x}(x-1)+1\right) \geq-\alpha$ for $x \leq 0$. Therefore

$$
\begin{aligned}
& \alpha M+(D K(1)-K(1))-\frac{1+\alpha}{\delta}((1-\delta) K(1)-K(1-\delta)) \\
& =\alpha M+\frac{1}{2} C(1-(1+\alpha)(1-\delta))+g_{\alpha}(x) * \nu \\
& \geq \alpha M+0+g_{\alpha}(x) 1_{[m, \infty)}(x) * \nu+g_{\alpha}(x) 1_{(-\infty, m)}(x) * \nu \\
& \geq \alpha M+0-\alpha M=0,
\end{aligned}
$$

which proves the claim.

Proposition 3.15 Let $\delta \in(0,1]$ and suppose that $\left|x e^{x}-h(x)\right| * \nu \in \mathcal{V}$. Then

$$
(D K(1)-K(1))-\frac{1}{\delta}((1-\delta) K(1)-K(1-\delta))
$$

is a non-negative increasing process.

Proof Step 1: Because of Proposition 3.13 we can replace $X$ with $X-K(1)$. Consequently, we may w.l.o.g. assume that $K(1)=\widetilde{K}(1)=0$ and $\widehat{W}(1)=0$.

Step 2: As in the proof of Proposition 3.14 it follows that $D \widetilde{K}(1)-\widetilde{K}(1)-$ $\frac{1}{\delta}((1-\delta) \widetilde{K}(1)-\widetilde{K}(1-\delta))=\frac{1}{2} C(1-(1-\delta))+g_{0}(x) * \nu$. Since $1-(1-\delta)=\delta>0$ and $g_{0} \geq 0$, we have that this process is increasing.

Step 3: Since (3.4) and $D \widetilde{K}(1)-\widetilde{K}(1)-\frac{1}{\delta}((1-\delta) \widetilde{K}(1)-\widetilde{K}(1-\delta))$ grow identically outside the thin set $\{\widehat{W}(1) \neq 0\} \cup\{\widehat{W}(1-\delta) \neq 0\}$ (i.e., the stochastic integral of $1_{\{\widehat{W}(1)=\widehat{W}(1-\delta)=0\}}$ with respect to both processes coincides), it remains to be shown that the jumps of (3.4) are non-negative. Since $\widehat{W}(1)=0$, we have $\Delta\left(D K(1)-K(1)+\frac{1}{\delta}((1-\delta) K(1)-K(1-\delta))\right)_{t}=\int\left((x-1) e^{x}+1\right) \nu(\{t\} \times$ $d x)+\frac{1}{\delta} \log \left(1+\int\left(e^{(1-\delta) x}-1\right) \nu(\{t\} \times d x)\right)$ (cf., Remark 3 following Proposition 2.25 and Theorem 2.18). This equals $\int x e^{x} Q(d x)+\frac{1}{\delta} \log \left(\int e^{(1-\delta) x} Q(d x)\right)$ if we define the probability measure $Q(\cdot):=\nu(\{t\} \times \cdot)+(1-\nu(\{t\} \times \mathbb{R})) \varepsilon_{0}(\cdot)$. Note that $\int e^{x} Q(d x)=1+\widehat{W}(1)=1$. Proposition 3.12 yields $\frac{1}{\delta} \log \left(\int e^{(1-\delta) x} Q(d x)\right) \geq$ $-\int x e^{x} Q(d x)$. Altogether, it follows that the jumps of (3.4) are non-negative.

Now we turn to the proof of the sufficient conditions for uniform integrability.

Proof of Proposition 3.3 The assertion follows immediately from JS, I.1.47 and Jacod (1979), Exercise 1.12.

Proof of Lemma 3.4 The claims follow from Doob's stopping theorem (cf., JS, I.1.39) and Proposition 3.11.

Lemma 3.16 Let $a \in[0,1), \delta \in\left(0, \frac{1}{a}-1\right)$. If Condition $I(a, 1+\delta)^{1+\varepsilon}$ holds, then $Z \in \mathcal{M}$. 
Proof W.1.o.g. $K(1)=0$ (cf., Proposition 3.13). Choose $\varepsilon>0$ as in the definition of Condition $I(a, 1+\delta)^{1+\varepsilon}$ and let $\widetilde{\varepsilon}:=\frac{\delta \varepsilon}{1+\delta+\varepsilon}$. Define $\alpha \in(1, \infty)$ by $a=\frac{1}{1+\alpha \delta}$. Note that $\exp ((1+\widetilde{\varepsilon}) X)=\exp \left(\left(1-\alpha^{-1}\right) \frac{1+\widetilde{\varepsilon}}{1+\delta}((1+\delta) X-K(1+\right.$ $\delta)) \exp \left(\alpha^{-1} \frac{1+\widetilde{\varepsilon}}{1+\delta}((1+\delta) X-K(1+\delta))+\frac{1+\widetilde{\varepsilon}}{1+\delta} K(1+\delta)\right)$. Application of Hölder's inequality with $\frac{1}{p}:=\left(1-\alpha^{-1}\right) \frac{1+\widetilde{\varepsilon}}{1+\delta}$ and $\frac{1}{q}:=1-\frac{1}{p}$ yields

$$
\begin{aligned}
& E\left(\exp \left((1+\widetilde{\varepsilon}) X_{T}\right)\right) \\
& \leq\left(E\left(\exp \left((1+\delta) X_{T}-K(1+\delta)_{T}\right)\right)\right)^{\frac{1}{p}} \\
& \quad\left(E \left(\operatorname { e x p } \left(\frac{1+\alpha \delta}{1+\alpha \delta(1+\varepsilon)^{-1}}\right.\right.\right. \\
& \left.\left.\left.\quad\left(a\left((1+\delta) X_{T}-K(1+\delta)_{T}\right)+\frac{1-a}{\delta} K(1+\delta)_{T}\right)\right)\right)\right)^{\frac{1}{q}}
\end{aligned}
$$

for any finite stopping time $T$. The first expectation on the right-hand side of Inequality (3.5) is bounded by 1 because $(1+\delta) X-K(1+\delta)$ is a positive supermartingale (cf., JS, I.1.39). Note that $\frac{1+\alpha \delta}{1+\alpha \delta(1+\varepsilon)^{-1}} \leq 1+\varepsilon$. Hence, Condition $I(a, 1+\delta)^{1+\varepsilon}$ implies that the second integral is bounded uniformly over all finite stopping times $T$. In view of Proposition 3.3, we are done.

Proof of Theorem 3.6 W.1.o.g. $a \neq 1, K(1)=0$ (cf., Propositions 3.3 and 3.13). Since $Z$ is a non-negative local martingale and hence a supermartingale, we only have to prove that $E\left(Z_{\infty}\right) \geq 1$.

Step 1: Choose $\delta>0$ so small that $a+\delta<1$ and set $\widetilde{\delta}:=\frac{1}{1-\delta}-1$. Let $Y:=(1-\delta) X$. In view of Proposition 3.15, straightforward calculations yield that $a\left((1+\widetilde{\delta}) Y-K^{Y}(1+\widetilde{\delta})\right)+\frac{1-a}{\widetilde{\delta}}\left(K^{Y}(1+\widetilde{\delta})-(1+\widetilde{\delta}) K^{Y}(1)\right) \leq a X+$ $(1-a) D K(1)$. Therefore, Condition $I(a, 1+\widetilde{\delta})^{1+\varepsilon}$ holds for $Y$ instead of $X$ and hence $\exp \left(Y-K^{Y}(1)\right) \in \mathcal{M}$ by Lemma 3.16.

Step 2: Since $\exp \left(Y-K^{Y}(1)\right)_{\infty}=Z_{\infty}^{\frac{1-a-\tilde{\delta} a}{(1+\tilde{\delta})(1-a)}} \liminf \inf _{t \rightarrow \infty} \exp \left(\frac{\widetilde{\delta} a}{(1+\widetilde{\delta})(1-a)}\right.$ $\left.X_{t}-K(1-\delta)_{t}\right)$, Step 1 and the application of Hölder's inequality with $\frac{1}{p}:=$ $\frac{1-a-\widetilde{\delta} a}{(1+\widetilde{\delta})(1-a)}$ and $\frac{1}{q}:=1-\frac{1}{p}=\frac{\delta}{1-a}$ yields

$$
1 \leq\left(E\left(Z_{\infty}\right)\right)^{\frac{1}{p}} \liminf _{t \rightarrow \infty}\left(E\left(\exp \left(a X_{t}-\frac{1-a}{\delta} K(1-\delta)_{t}\right)\right)\right)^{\frac{1}{q}}
$$

Since $\frac{1}{p} \rightarrow 1$ for $\delta \rightarrow 0$, it remains to be shown that the second factor converges to 1 for $\delta \rightarrow 0$.

Step 3: By Proposition 3.15, we have $-\frac{1}{\delta} K(1-\delta) \leq D K(1)$. Since Condition $I(a, 1)^{1+\varepsilon}$ implies $I(a, 1)$, it follows that $E\left(\exp \left(a X_{t}-\frac{1-a}{\delta} K(1-\delta)_{t}\right)\right) \leq$ $E\left(\exp \left(a X_{t}+(1-a) D K(1)_{t}\right)\right)$ is bounded uniformly in $t$. Since $\frac{1}{q} \rightarrow 0$ for 
$\delta \rightarrow 0$, this implies that the second factor on the right-hand side of Inequality (3.6) converges to 1 as desired.

Proof of Theorem 3.7 W.l.o.g. $K(1)=0$ (cf., Proposition 3.13).

Step 1: Define $\delta, \widetilde{\delta}$ and $Y$ as in Step 1 of the proof of Theorem 3.6. Applying Proposition 3.14 instead of Proposition 3.15 in that proof, we have $a((1+\widetilde{\delta}) Y-$ $\left.K^{Y}(1+\widetilde{\delta})\right)+\frac{1-a}{\widetilde{\delta}}\left(K^{Y}(1+\widetilde{\delta})-(1+\widetilde{\delta}) K^{Y}(1)\right) \leq a X+\frac{1-a}{1+\alpha} D K(1)+\frac{\alpha}{1+\alpha} M$ for some $\alpha>0$. Choose $\varepsilon>0$ so small that $1+\varepsilon \leq \frac{1+\alpha}{1+\alpha a}$ and set $\widetilde{a}:=a(1+\varepsilon)$. It follows that $(1+\varepsilon)\left(\left(a(1+\widetilde{\delta}) Y-K^{Y}(1+\widetilde{\delta})\right)+\frac{1-a}{\widetilde{\delta}}\left(K^{Y}(1+\widetilde{\delta})-(1+\widetilde{\delta}) K^{Y}(1)\right)\right) \leq$ $\widetilde{a} X+(1-\widetilde{a}) D K(1)+\alpha M$. Hence Condition $I(\widetilde{a}, 1+\widetilde{\delta})^{1+\varepsilon}$ holds for $Y$ instead of $X$, which implies that $\exp \left(Y-K^{Y}(1)\right) \in \mathcal{M}$ by Lemma 3.16.

Step 2: $Z \in \mathcal{M}$ follows now as in Steps 2 and 3 of the proof of Theorem 3.6.

Lemma 3.17 Let $\delta \in(0,1)$. If Condition $I(0,1+\delta)$ holds, then $Z \in \mathcal{M}$.

Proof W.1.o.g. $K(1)=0$ (cf., Proposition 3.13). Since $Z$ is a non-negative local martingale and hence a supermartingale, we only have to prove that $E\left(Z_{\infty}\right) \geq 1$.

Step 1: Define the local martingale $\widetilde{Z}:=\exp ((1+\delta) X-K(1+\delta))$. By JS, I.3.10 there exists a sequence of stopping times $\left(S_{n}\right)_{n \in \mathbb{N}}$ with $S_{n} \uparrow \propto P$-almost surely and $K(1+\delta)_{t} \leq n$ on $\left[0, S_{n}\right]$. Fix $n \in \mathbb{N}$. Since $\widetilde{Z}$ is a supermartingale, we have $E\left(\left(Z^{S_{n}}\right)_{T}^{1+\delta}\right)=E\left(\widetilde{Z}_{S_{n} \wedge T} \exp \left(K(1+\delta)_{S_{n} \wedge T}\right)\right) \leq e^{n}$ for any finite stopping time T. By Jacod (1979), Exercise (1.12) and JS, I.1.47, this implies that $Z^{S_{n}}$ is a uniformly integrable martingale.

Step 2: For $n \in \mathbb{N}$ and $t \in \mathbb{R}_{+}$we have $Z_{S_{n} \wedge t} 1_{\left\{S_{n}<\infty\right\}}=\widetilde{Z}_{S_{n} \wedge t}^{(1+\delta)^{-1}} \exp \left(\frac{1}{1+\delta}\right.$ $\left.K(1+\delta)_{S_{n} \wedge t}\right) 1_{\left\{S_{n}<\infty\right\}}$. Application of Hölder's inequality with $\frac{1}{p}:=\frac{1}{1+\delta}$ and $\frac{1}{q}:=1-\frac{1}{p}=\frac{\delta}{1+\delta}$ yields

$$
\begin{aligned}
& \sup _{t \in \mathbb{R}_{+}} E\left(Z_{S_{n} \wedge t} 1_{\left\{S_{n}<\infty\right\}}\right) \leq\left(\sup _{t \in \mathbb{R}_{+}} E\left(\widetilde{Z}_{S_{n} \wedge t}\right)\right)^{\frac{1}{p}} \\
& \left(\sup _{t \in \mathbb{R}_{+}} E\left(\exp \left(\frac{1}{\delta} K(1+\delta)_{S_{n} \wedge t}\right) 1_{\left\{S_{n}<\infty\right\}}\right)\right)^{\frac{1}{q}} .
\end{aligned}
$$

The first factor on the right-hand side is dominated by 1 because $\widetilde{Z}$ is a supermartingale. From Theorem 2.18 it follows that $K(1+\delta)=K(1+\delta)-(1+\delta) K(1)$ is increasing. Condition $I(0,1+\delta)$ implies that $P\left(S_{n}<\infty\right) \rightarrow 0$ for $n \rightarrow \infty$. Dominated convergence and once more Condition $I(0,1+\delta)$ yields that the second factor on the right-hand side of Inequality (3.7) converges to 0 for $n \rightarrow \infty$. From Fatou's lemma we conclude that $E\left(Z_{S_{n}} 1_{\left\{S_{n}<\infty\right\}}\right) \rightarrow 0$ for $n \rightarrow \infty$. Since $E\left(Z_{\infty}\right)=$ $E\left(Z_{\infty}^{S_{n}}\right)+E\left(Z_{\infty} 1_{\left\{S_{n}<\infty\right\}}\right)-E\left(Z_{S_{n}} 1_{\left\{S_{n}<\infty\right\}}\right) \geq 1-E\left(Z_{S_{n} \wedge t} 1_{\left\{S_{n}<\infty\right\}}\right)$, we obtain $E\left(Z_{\infty}\right)=1$.

Proposition 3.18 Condition $I(0,1)$ implies Condition $I(0,1-)$.

Proof This follows from Proposition 3.15. 
Lemma 3.19 If Condition $I(0,1-)$ holds, then $Z \in \mathcal{M}$.

Proof W.l.o.g. $K(1)=0$ (cf., Proposition 3.13). Since $Z$ is a non-negative local martingale and hence a supermartingale, we only have to prove that $E\left(Z_{\infty}\right) \geq 1$.

Step 1: Choose $\delta \in(0,1)$ so small that $\sup _{t \in \mathbb{R}_{+}} E\left(\exp \left(-\frac{1}{\delta} K(1-\delta)_{t}\right)\right)<\infty$. Let $Y:=(1-\delta) X$ and $\widetilde{\delta}:=\frac{1}{1-\delta}-1$. It follows that $\frac{1}{\delta}\left(K^{Y}(1+\widetilde{\delta})-(1+\right.$ $\left.\widetilde{\delta}) K^{Y}(1)\right)=-\frac{1}{\delta} K(1-\delta)$. Therefore Condition $I(0,1+\delta)$ holds for $Y$ instead of $X$, which implies that $\exp \left(Y-K^{Y}(1)\right) \in \mathcal{M}$ by Lemma 3.17.

Step 2: Since $\exp \left(Y-K^{Y}(1)\right)=Z^{1-\delta} \exp (-K(1-\delta))$, Step 1 and Hölder's inequality with $\frac{1}{p}:=1-\delta$ and $\frac{1}{q}:=1-\frac{1}{p}=\delta$ yield that

$1=E\left(\exp \left(Y-K^{Y}(1)\right)_{\infty}\right) \leq\left(E\left(Z_{\infty}\right)\right)^{1-\delta}\left(E\left(\exp \left(-\frac{1}{\delta} K(1-\delta)_{\infty}\right)\right)\right)^{\delta}$.

Note that the second factor on the right-hand side converges to 1 for $\delta \rightarrow 0$ by Condition $I(0,1-)$. Since $1-\delta \rightarrow 1$ for $\delta \rightarrow 0$, it follows that $E\left(Z_{\infty}\right) \geq 1$ as desired.

Proof of Theorem 3.2 This follows from Proposition 3.18 and Lemma 3.19.

Proof of Corollary 3.10 Theorems 3.2, 3.6, 3.7

\section{Esscher's change of measure in finance}

If a probability space $(\Omega, \mathcal{F}, P)$ and a random variable $X$ are given, probability measures $P_{\vartheta}$ with Radon-Nikodým density $\frac{d P_{\vartheta}}{d P}:=\exp (\vartheta X-k(\vartheta))$ for some real numbers $\vartheta, k(\vartheta)$ are called Esscher transform because they were applied by Esscher in the actuaries (cf., Esscher 1932). This concept has been transferred to finance by Gerber and Shiu (1994) and others. Suppose that $X$ is a real-valued Lévy process. If $k(\vartheta)$ denotes the cumulant of $X$, then $Z^{\vartheta}:=\left(\exp \left(\vartheta X_{t}-t k(\vartheta)\right)\right)_{t \in \mathbb{R}_{+}}$ is the density process of some probability measure $P_{\vartheta} \stackrel{\text { loc }}{\sim} P$ for any $\vartheta \in \mathbb{R}$. In finance one is particularly interested in so-called equivalent martingale measures, i.e. measures $P^{*} \sim P$ such that some given security price process $S$ becomes a martingale or at least a local martingale.

Fix a terminal time $T \in \mathbb{R}_{+}$and suppose that $S$ is of the form $S=S_{0} \exp (X)$. Then a necessary and sufficient condition for $\left(S_{t}\right)_{t \in[0, T]}$ to be a $P_{\vartheta}$-martingale is

$$
k(\vartheta+1)-k(\vartheta)=0
$$

(cf., Shiryaev 1999, VII.3c). This kind of measure change has been considered by Madan and Milne (1991), Gerber and Shiu (1994), Eberlein and Keller (1995). A closely related security model is $S=S_{0} \mathcal{E}(X)$ if $\Delta X>-1$. In fact, any such positive process $S=S_{0} \mathcal{E}(X)$ can be written as $S=S_{0} \exp (\widetilde{X})$ for some other Lévy process $\widetilde{X}$ and vice versa (cf., Goll and Kallsen 2000, Lemma A.8). In this case, a necessary and sufficient condition for $\left(S_{t}\right)_{t \in[0, T]}$ to be a $P_{\vartheta}$-martingale is

$$
k^{\prime}(\vartheta)=0 .
$$


The corresponding measure $P_{\vartheta}$ minimizes the relative entropy among all equivalent martingale measures (cf., Miyahara 1999; Chan 1999) and it can be used to determine optimal portfolios relative to exponential utility (cf., Kallsen 2000). Note that the two measure transformations above generally differ although they correspond to the same class of security price models. Whereas Gerber and Shiu (1994) used the notion Esscher transform in the first case, Chan (1999) applied the same name to the second setting.

In this section we want to extend this approach to general semimartingales. More specifically, suppose that $X$ is a $\mathbb{R}^{d}$-valued semimartingale whose characteristics are of the form (2.1). For any $\vartheta \in L(X)$ such that $\vartheta^{\top} \cdot X$ is exponentially special, define a local martingale

$$
Z^{\vartheta}:=\exp \left(\vartheta^{\top} \cdot X-K^{X}(\vartheta)\right) .
$$

If it is a uniformly integrable martingale (cf., the previous section), then it is the density process of some probability measure $P_{\vartheta} \sim P$. As in the Lévy process setting, we want to determine necessary and sufficient conditions for some $\mathbb{R}^{d}$ valued securities price process $S=\left(S^{1}, \ldots, S^{d}\right)$ to be a $P_{\vartheta}$-local martingale.

Firstly, let us consider the case

$$
S^{i}=S_{0}^{i} \exp \left(X^{i}\right) \text { for } i=1, \ldots, d
$$

with $S_{0}^{i} \in \mathbb{R} \backslash\{0\}$.

Theorem 4.1 Let $\vartheta \in L(X)$ be such that $\vartheta^{\top} \cdot X$ is exponentially special and such that $Z^{\vartheta}$ is a uniformly integrable martingale. Define $P_{\vartheta} \sim P$ by its Radon-Nikodým density $\frac{d P_{\vartheta}}{d P}:=Z_{T}^{\vartheta}$ and set $\vartheta^{(i)}:=\left(\vartheta^{1}, \ldots, \vartheta^{i-1}, \vartheta^{i}+1, \vartheta^{i+1}, \ldots, \vartheta^{d}\right)^{\top}$. Then the processes $S^{i}=S_{0}^{i} \exp \left(X^{i}\right)$ are $P_{\vartheta}$-local martingales if and only if $\left(\vartheta^{(i)}\right)^{\top} \cdot X$ is exponentially special and

$$
K^{X}\left(\vartheta^{(i)}\right)-K^{X}(\vartheta)=0 \text { for } i=1, \ldots, d .
$$

In this case we call $P_{\vartheta}$ an Esscher martingale transform for exponential processes.

Proof By JS, III.3.8, $\exp \left(X^{i}\right)$ is a $P_{\vartheta}$-local martingale if and only if $\exp \left(X^{i}\right) Z^{\vartheta}=$ $\exp \left(\left(\vartheta^{(i)}\right)^{\top} \cdot X-K^{X}(\vartheta)\right)$ is a $P_{\vartheta}$-local martingale. By Lemma 2.15 and Theorem 2.19 this is the case if and only if $\left(\vartheta^{(i)}\right)^{\top} \cdot X$ is exponentially special and $K^{X}\left(\vartheta^{(i)}\right)=K^{X}(\vartheta)$ up to indistinguishability.

Theorem 4.2 If $d=1$, then the Esscher martingale transform for exponential processes is unique (provided that it exists).

Proof Step 1: Let $\vartheta, \bar{\vartheta} \in L(X)$ be such that $\vartheta \cdot X,(\vartheta+1) \cdot X, \bar{\vartheta} \cdot X,(\bar{\vartheta}+1) \cdot X$ are exponentially special and such that $P_{\vartheta}, P_{\bar{\vartheta}}$ are Esscher martingale transforms for exponential processes. Then $K^{X}(\vartheta+1)-K^{X}(\vartheta)=0=K^{X}(\bar{\vartheta}+1)-K^{X}(\bar{\vartheta})$ (cf., Theorem 4.1). In particular, $\widetilde{\kappa}(\vartheta+1)-\widetilde{\kappa}(\vartheta)=0=\widetilde{\kappa}(\bar{\vartheta}+1)-\widetilde{\kappa}(\bar{\vartheta})(P \otimes A)$ almost everywhere on the set $\{\Delta A=0\}$ (cf., Theorem 2.18). On the set $\{\Delta A \neq 0\}$ we have $\Delta K^{X}(\vartheta+1)-\Delta K^{X}(\vartheta)=0=\Delta K^{X}(\bar{\vartheta}+1)-\Delta K^{X}(\bar{\vartheta})$. In view of Theorem 2.18, this implies $0=b+\frac{1}{2} c+c \vartheta+\int\left(\left(e^{x}-1\right) e^{\vartheta x}-h(x)\right) F(d x)$ 
on the set $\{\Delta A=0\}$ and $0=\log \frac{1+\int\left(e^{(\vartheta+1) x}-1\right) \nu(\{t\} \times d x)}{1+\int\left(e^{\vartheta x}-1\right) \nu(\{t\} \times d x)}$ on the set $\{\Delta A \neq 0\}$. Parallel statements hold for $\bar{\vartheta}$.

Step 2: Fix $(\omega, t) \in \Omega \times[0, T]$ and let $\vartheta \leq \bar{\vartheta}$ in $(\omega, t)$ w.l.o.g. Firstly, suppose that $\Delta A_{t}(\omega)=0$. Since $\int\left|\left(e^{x}-1\right) e^{\vartheta x}-h(x)\right| F_{t}(d x)<\infty$ and likewise for $\bar{\vartheta}$, we have that $\sup _{\psi \in[\vartheta, \bar{\vartheta}]} \int\left|\left(e^{x}-1\right) e^{\psi x}-h(x)\right| F_{t}(d x)<\infty$. Define $v:[0,1] \rightarrow \mathbb{R}$ by $v(\lambda):=b_{t}+\frac{1}{2} c_{t}+c_{t}(\vartheta+\lambda(\bar{\vartheta}-\vartheta))+\int\left(\left(e^{x}-1\right) e^{(\vartheta+\lambda(\bar{\vartheta}-\vartheta)) x}-h(x)\right) F_{t}(d x)$. Note that $v$ is a well-defined, continuous, increasing mapping. Similarly as in Step 2 of the proof of Theorem 4.5 below one concludes that $(\bar{\vartheta}-\vartheta) c_{t}=0,(\bar{\vartheta}-\vartheta) b_{t}-$ $\int(\bar{\vartheta}-\vartheta) h(x) F_{t}(d x)=0$, and $(\bar{\vartheta}-\vartheta) x=0$ for $F_{t}$-almost all $x \in \mathbb{R}$.

Secondly, assume that $\Delta A_{t}(\omega) \neq 0$. Since $\int e^{(\vartheta+1) x} \nu(\{t\} \times d x)<\infty$ and $\int e^{\vartheta x} \nu(\{t\} \times d x)<\infty$ and likewise for $\bar{\vartheta}$, the same integrability conditions hold uniformly on $[\vartheta, \bar{\vartheta}]$. This time, define $v:[0,1] \rightarrow \mathbb{R}$ by $v(\lambda):=$ $\log \frac{1+\int\left(e^{(\vartheta+\lambda(\bar{\vartheta}-\vartheta)+1) x}-1\right) \nu(\{t\} \times d x)}{1+\int\left(e^{(\vartheta+\lambda(\bar{\vartheta}-\vartheta)) x}-1\right) \nu(\{t\} \times d x)}$. Observe that $v$ is differentiable on $(0,1)$ with derivative

$$
\begin{aligned}
v^{\prime}(\lambda)= & \frac{(\bar{\vartheta}-\vartheta) \int x e^{(\vartheta+\lambda(\bar{\vartheta}-\vartheta)) x} e^{x} \nu(\{t\} \times d x)}{1+\int\left(e^{(\vartheta+\lambda(\bar{\vartheta}-\vartheta)) x} e^{x}-1\right) \nu(\{t\} \times d x)} \\
& -\frac{(\bar{\vartheta}-\vartheta) \int x e^{(\vartheta+\lambda(\bar{\vartheta}-\vartheta)) x} \nu(\{t\} \times d x)}{1+\int\left(e^{(\vartheta+\lambda(\bar{\vartheta}-\vartheta)) x}-1\right) \nu(\{t\} \times d x)} .
\end{aligned}
$$

Fix $\lambda \in(0,1)$ for the moment. Define a family $\left(Q_{\varrho}\right)_{\varrho \in[0,1]}$ of probability measures on $\mathbb{R}$ by $Q_{\varrho}(M):=\left(\int e^{\varrho x} e^{(\vartheta+\lambda(\bar{\vartheta}-\vartheta)) x} P^{\Delta X_{t} \mid \mathcal{F}_{t-}}(d x)\right)^{-1} \int_{M} e^{\varrho x} e^{(\vartheta+\lambda(\bar{\vartheta}-\vartheta)) x}$ $P^{\Delta X_{t} \mid \mathcal{F}_{t-}}(d x)$ for $M \in \mathcal{B}$. With this notion, we have $v^{\prime}(\lambda)=(\bar{\vartheta}-\vartheta)\left(E_{Q_{1}}(I)-\right.$ $E_{Q_{0}}(I)$ ), where $I: \mathbb{R} \rightarrow \mathbb{R}, x \rightarrow x$ denotes the identity mapping (cf., JS, II.1.26). Since $\left(Q_{\varrho}\right)_{\varrho \in[0,1]}$ is a class with increasing likelihood ratio, it follows that $v^{\prime}(\lambda) \geq 0$ (cf., Witting 1985, Satz 2.28). Therefore $v$ is an increasing mapping on $[0,1]$. Similarly as in Step 2 of the proof of Theorem 4.5 below we conclude that $v(\lambda)=0$ and $v^{\prime}(\lambda)=0$ for any $\lambda \in(0,1)$. This implies $0=v^{\prime}(\lambda)=$ $(\bar{\vartheta}-\vartheta) \int x e^{(\vartheta+\lambda(\bar{\vartheta}-\vartheta)) x}\left(e^{x}-1\right) \nu(\{t\} \times d x)$ for any $\lambda \in(0,1)$, which in turn means that $(\bar{\vartheta}-\vartheta) x=0$ for $\nu(\{t\} \times \cdot)$-almost all $x \in \mathbb{R}$.

Step 3: As in the proof of Theorem 4.5 below it follows that $\vartheta \cdot X=\bar{\vartheta} \cdot X$, which proves the claim.

Uniqueness may cease to hold in markets with more than one underlying:

Example 4.3 We consider a simple one-period model with $T:=1$ and $\mathcal{F}_{t}=$ $\{\varnothing, \Omega\}$ for $t \in[0,1)$. The sigma-field $\mathcal{F}=\mathcal{F}_{1}$ is supposed to be generated by some random variable $U$ with $P(U=-1)=P(U=0)=P(U=1)=\frac{1}{3}$. Define securities price processes $S^{1}, S^{2}$ by $S_{t}^{1}=1$ for $t \in[0,1), S_{1}^{1}=e^{U}$, and $S^{2}:=1+S^{1}$. Moreover, define probability measures $P^{\prime}, P^{\prime \prime} \sim P$ via $\frac{d P^{\prime}}{d P}:=$ $\frac{1}{c^{\prime}} \exp \left(-\frac{1}{2} U\right)$ for $c^{\prime}:=E\left(\exp \left(-\frac{1}{2} U\right)\right)$ and $\frac{d P^{\prime \prime}}{d P}:=\frac{1}{c^{\prime \prime}} \frac{2}{1+e^{U}}$ for $c^{\prime \prime}:=E\left(\frac{2}{1+e^{U}}\right)$. A straightforward calculation yields that $S^{1}, S^{2}$ are martingales relative to both $P^{\prime}$ and $P^{\prime \prime}$. If we set $X^{1}:=\log \left(S^{1}\right)$ and $X^{2}:=\log \left(S^{2}\right)$, then $\frac{d P^{\prime}}{d P}=\exp \left(-\frac{1}{2}\left(X_{1}^{1}-\right.\right.$ $\left.\left.X_{0}^{1}\right)-K_{1}^{X^{1}}\left(-\frac{1}{2}\right)\right)$ and $\frac{d P^{\prime \prime}}{d P}=\exp \left(-\left(X_{1}^{2}-X_{0}^{2}\right)-K_{1}^{X^{2}}(-1)\right)$. Therefore both 
measures are Esscher martingale transforms for exponential processes, namely corresponding to $\vartheta=\left(-\frac{1}{2}, 0\right)$ and $\vartheta=(0,-1)$, respectively.

The following theorems correspond to the securities price process

$$
S^{i}=S_{0}^{i} \mathcal{E}\left(X^{i}\right) \text { for } i=1, \ldots, d,
$$

where we assume $\Delta X^{i} \neq-1$ outside some evanescent set and $S_{0}^{i} \in \mathbb{R} \backslash\{0\}$. Note that $S^{i}$ is a local martingale if and only if $X^{i}$ is a local martingale because $\frac{1}{S_{-}^{i}}$ is locally bounded. Therefore one can apply the following result also to the model

$$
S^{i}=X^{i} \text { for } i=1, \ldots, d .
$$

Theorem 4.4 Let $\vartheta \in L(X)$ be such that $\vartheta^{\top} \cdot X$ is exponentially special and such that $Z^{\vartheta}$ is a uniformly integrable martingale. Define $P_{\vartheta} \sim P$ by its Radon-Nikodým density $\frac{d P_{\vartheta}}{d P}:=Z_{T}^{\vartheta}$. Then the processes $S^{i}=S_{0}^{i} \mathcal{E}\left(X^{i}\right)$ are $P_{\vartheta}$-local martingales if and only if $\left|x^{i} e^{\vartheta^{\top} x}-h^{i}(x)\right| * \nu \in \mathcal{V}$ for $i=1, \ldots, d$ and

$$
D K^{X}(\vartheta)=0 \text {. }
$$

In this case we call $P_{\vartheta}$ an Esscher martingale transform for linear processes.

Proof “ $\Rightarrow$ ": Suppose that $S^{i}$, or equivalently, $X^{i}$ is a $P_{\vartheta}$-local martingale for $i=1, \ldots, d$. By Corollary 2.21, the $P_{\vartheta}$-characteristics $(\bar{B}, \bar{C}, \bar{\nu})$ of $X$ are of the form (2.1) but with $\bar{b}=b+c \vartheta+\int h(x)\left(\frac{e^{\vartheta^{\top} x}}{1+\widehat{W}(\vartheta)}-1\right) F(d x), \bar{c}=c, \bar{F}(d x)=$ $\frac{e^{\vartheta^{\top} x}}{1+\widehat{W}(\vartheta)} F(d x)$ instead of $(b, c, F)$. Since $X^{i}$ is a $P_{\vartheta}$-local martingale, we have $0=\bar{B}^{i}+\left(x^{i}-h^{i}(x)\right) * \bar{\nu}=B^{i}+\vartheta^{\top} \cdot C^{\cdot i}+\left(x^{i} \frac{e^{\vartheta^{\top} x}}{1+\widehat{W}(\vartheta)}-h^{i}(x)\right) * \nu$ for $i=1, \ldots, d$ (cf., Lemma 2.11). In particular, we have $\left|x^{i} \frac{e^{\vartheta^{\top} x}}{1+\widehat{W}(\vartheta)}-h^{i}(x)\right| * \nu \in \mathcal{V}$. As in the proof of Proposition 2.25, it follows that $\left(\frac{\widehat{W}(\vartheta)}{1+\widehat{W}(\vartheta)} h^{i}(x)\right) * \nu=\frac{\widehat{W}(\vartheta)}{1+\widehat{W}(\vartheta)} \cdot B^{i} \in$ $\mathcal{V}$. Together, we have that $\left|(1+\widehat{W}(\vartheta))^{-1}\left(x^{i} e^{\vartheta^{\top} x}-h^{i}(x)\right)\right| * \nu \in \mathcal{V}$. Since $1+\widehat{W}(\vartheta)$ is locally bounded (cf., e.g., Statement 4 in Theorem 2.18), it follows that $\left|x^{i} e^{\vartheta^{\top} x}-h^{i}(x)\right| * \nu \in \mathcal{V}$ as well (cf., JS, I.3.5). Proposition 2.25 yields that $D_{i} K^{X}(\vartheta)=0$ for $i=1, \ldots, d$.

" $\Leftarrow$ ”: Conversely, assume that $D K^{X}(\vartheta)=0$. From Proposition 2.25, we conclude that $0=b^{i}+c^{i \cdot} \vartheta+\int\left(\frac{x^{i} e^{\vartheta^{\top} x}}{1+\widehat{W}(\vartheta)}-h^{i}(x)\right) F(d x)=\bar{b}^{i}+\int\left(x^{i}-h^{i}(x)\right) \bar{F}(d x)$ for $i=1, \ldots, d$, where $\bar{b}, \bar{c}, \bar{F}$ are defined as in Step 1. In view of Lemma 2.11, we are done.

Theorem 4.5 The Esscher martingale transform for linear processes is unique (provided that it exists).

Proof Step 1: Let $\vartheta, \bar{\vartheta} \in L(X)$ be such that $\vartheta^{\top} \cdot X, \bar{\vartheta}^{\top} \cdot X$ are exponentially special and such that $P_{\vartheta}, P_{\bar{\vartheta}}$ are Esscher martingale transforms for linear processes. Then $D K^{X}(\vartheta)=0=D K^{X}(\bar{\vartheta})$ (cf., Theorem 4.4) and hence $D_{i} \widetilde{K}^{X}(\vartheta)=$ 
$(1+\widehat{W}(\vartheta)) \cdot D_{i} K^{X}(\vartheta)=0$ for $i=1, \ldots, d$ and likeweise for $\bar{\vartheta}$. In particular, we have $D_{i} \widetilde{\kappa}(\vartheta)=0=D_{i} \widetilde{\kappa}(\bar{\vartheta})(P \otimes A)$-almost everywhere for $i=1, \ldots, d$.

Step 2: Fix $(\omega, t) \in \Omega \times[0, T]$. Suppose that $\vartheta \leq \bar{\vartheta}$ in $(\omega, t)$. Since $\int \mid x e^{\vartheta^{\top} x}-$ $h(x) \mid F_{t}(d x)<\infty$ and likewise for $\bar{\vartheta}$, we have that $\sup _{\lambda \in[0,1]} \int \mid x e^{(\vartheta+\lambda(\bar{\vartheta}-\vartheta))^{\top} x}-$ $h(x) \mid F_{t}(d x)<\infty$. Define $v:[0,1] \rightarrow \mathbb{R}$ by $v(\lambda):=(\bar{\vartheta}-\vartheta)^{\top}\left(b_{t}+c_{t}(\vartheta+\right.$ $\left.\lambda(\bar{\vartheta}-\vartheta))+\int\left(x e^{(\vartheta+\lambda(\bar{\vartheta}-\vartheta))^{\top} x}-h(x)\right) F_{t}(d x)\right)$. Moreover, set $u:[0,1] \rightarrow \mathbb{R}$, $\lambda \mapsto \int_{0}^{\lambda} v(z) d z$. Since $v$ is increasing, we have that $u$ is a closed proper convex mapping (Rockafellar 1970, Theorem 24.2). Let us identify $u$ with a convex function $\mathbb{R} \rightarrow \mathbb{R} \cup\{\infty\}$ by setting $u(\lambda):=\infty$ for $\lambda \notin[0,1]$. Since $u^{\prime}(\lambda)=v(\lambda)$ for $\lambda \in(0,1)$, Rockafellar (1970), Theorem 25.6 yields that $v(\lambda)$ belongs to the subdifferential $\partial u(\lambda)$ for any $\lambda \in[0,1]$. Step 1 implies that $v(0)=0=v(1)$, which means that $u$ achieves its infimum in 0 and 1 . Since the minimum set of a convex function is convex, we have that $v(\lambda)=0$ for any $\lambda \in[0,1]$. This implies that $(\bar{\vartheta}-\vartheta)^{\top} c_{t}(\bar{\vartheta}-\vartheta)=0$ and $(\bar{\vartheta}-\vartheta)^{\top} x=0$ for $F_{t}$-almost all $x \in \mathbb{R}^{d}$. This in turn yields $(\bar{\vartheta}-\vartheta)^{\top} c_{t}=0$ and hence $(\bar{\vartheta}-\vartheta)^{\top} b_{t}-\int(\bar{\vartheta}-\vartheta)^{\top} h(x) F_{t}(d x)=$ $v(0)=0$. From Kallsen and Shiryaev (2000), Lemma 2.5 we conclude that the characteristics of $(\bar{\vartheta}-\vartheta)^{\top} \cdot X$ vanish, which in turn means that $\vartheta^{\top} \cdot X=\bar{\vartheta}^{\top} \cdot X$ up to indistinguishability. This proves the assertion.

\section{Remarks}

1. Note that the conditions in both Theorems 4.1 and 4.4 correspond to $d$ equations in $d$ unknowns $\vartheta^{1}(\omega, t), \ldots, \vartheta^{d}(\omega, t)$ for fixed $(\omega, t)$.

2. If one is interested in the $P_{\vartheta}$-local martingale property of $X^{i}$ rather than $S^{i}$, then of course the restriction $\Delta X^{i} \neq-1$ is not necessary for Theorem 4.4 to hold.

3. Suppose that $X$ is a Lévy process. For real-valued Lévy processes $L$ it is wellknown that $e^{L}$ is a martingale if and only if it is a local martingale (cf., e.g., Kallsen (2000), Lemma 4.4). Consequently, $Z^{\vartheta}$ in Theorems 4.1 and 4.4 is automatically a martingale (and hence a uniformly integrable martingale if we restrict the time to some interval $[0, T]$ with $T \in \mathbb{R}_{+}$). Note that $X$ is still a Lévy process under the new measure $P_{\vartheta}$ as can be seen from the change of its characteristics (cf., Corollary 2.21 and JS, II.4.19). Therefore, the processes $S^{i}$ in Theorems 4.1 and 4.4 are $P_{\vartheta}$-martingales if they are positive $P_{\vartheta}$-local martingales (cf., e.g., Kallsen 2000, Lemmas 4.4 and 4.2).

4. Bühlmann et al. (1996) considered Esscher transforms in the sense of Equation (4.1) and Theorem 4.1 for general discrete-time processes. Their Equation (76) corresponds to Equation (4.2) above. Delbaen and Haezendonck (1989) characterize measure changes such that a compound Poisson process $X$ remains a compound Poisson process under the new probability measure. If the function $\beta$ in that paper is chosen as $x \mapsto \vartheta x$ for some $\vartheta \in \mathbb{R}$, one obtains the measure change $\frac{d P_{\vartheta}}{d P}=\exp \left(\vartheta X_{T}-K^{X}(\vartheta)_{T}\right)$ in our notation. Note, however, that the Esscher principle in Delbaen and Haezendonck (1989), Example 3.3 corresponds to a different class of measures. 
Finally, we want to consider the meaning of the two measures $P_{\vartheta}$ in Theorems 4.1 and 4.4 in finance on an informal level. Recall that arbitrage arguments do not suffice to determine unique derivative prices in incomplete markets. By contrast, even in popular stock price models the whole trivial arbitrage interval for European call option values can be obtained via expectation relative to some equivalent martingale measure (EMM) (cf., Eberlein and Jacod 1997; Frey and Sin 1999; Cvitanić et al. 1999). Additional criteria based on equilibrium-type arguments (e.g., Davis 1997; Karatzas and Kou 1996; Kallsen 2001, 2002), distance minimization (e.g., Keller 1997; Miyahara 1996, 1999; Chan 1999; Grandits 1999a,b; Fritelli 2000), and hedging arguments (e.g., Schäl 1994; Schweizer 1996) have been proposed to justify the choice of a particular EMM. Piecing together results from He and Pearson (1991a,b), Karatzas et al. (1991), Karamkov and Schachermayer (1999), Bellini and Frittelli (2000), Kallsen (1998), Schachermayer (2001), Goll and Rüschendorf (2001), Xia and Yan (2000), Delbaen et al. (2000) one can observe that these criteria are closely linked with each other as well as with portfolio optimization problems (cf., Kallsen 2001 for an overview). Fix a utility function $u$, a securities price process $S$, and a terminal time $T$. Very roughly speaking, an EMM with Radon-Nikodým density of the form $\frac{d P^{*}}{d P}=c u^{\prime}\left(x+\varphi^{\top} \cdot S_{T}\right)$ (for some $c \in \mathbb{R}_{+}, x \in \mathbb{R}, \varphi \in L(S)$ ) plays a threefold role in finance. Firstly, it leads to neutral contingent claim prices in the sense of Davis (1997), Kallsen (2001), i.e. utility maximizers do not trade options at these prices. Put differently, it corresponds to the least favourable market completion in the eyes of a utility maximizer. Secondly, it minimizes a certain distance functional among all EMM (e.g., the relative entropy in case of exponential utility). Thirdly, $\varphi$ is the expected utility maximizing portfolio relative to $u$ and initial capital $x$. Let us stress, however, that the extent to which these relationships hold in general settings depends sensitively on the chosen sets of trading strategies and probability measures, cf., Schachermayer (2000) for a thorough discussion and illuminating counterexamples.

How do Esscher transforms fit into this picture? As noted before, the EMM $P_{\vartheta}$ in a one-dimensional Lévy process setting of type (4.3) minimizes the relative entropy and it is related to exponential utility. On the above intuitive level, this is due to the fact that $\frac{d P_{\vartheta}}{d P}$ equals $\exp \left(\frac{\vartheta}{S_{-}} \cdot S_{T}\right)$ up to a multiplicative constant $\exp (-T k(\vartheta))$. If we leave the framework of Lévy processes, this is no longer true since the corresponding factor $\exp \left(-K^{X}(\vartheta)_{T}\right)$ is generally not a constant. However, $P_{\vartheta}$ can still be interpreted economically. It leads to neutral derivative prices for local utility in the sense of Kallsen $(2001,2002)$ if $u(x)=1-\exp (-x)$ is chosen as utility function.

The Esscher transform $P_{\vartheta}$ referring to real-valued Lévy processes of type (4.1) has a density proportionate to $S_{T}^{\vartheta}$. Therefore, it corresponds to the utility function $u(x)=x^{\vartheta+1}$ in the sense of the above overview (cf., also Naik and Lee 1990). Note, however, that this utility function depends on the solution parameter $\vartheta$. Moreover, the correspondence ceases to hold even for multidimensional Lévy processes.

An entirely mathematical property of Esscher transforms is that they can be computed relatively easily for general semimartingales because the whole density process is known in a form that is suitable to apply Girsanov's theorem (cf., Corollary 2.21). The unknown parameters ensuring the martingale property of the 
securities price process can be obtained by solving equations seperately for any $(\omega, t)$ (cf., Equations (4.2) and (4.4)). There exist further instances of such local measure changes which all share the property that they can be determined by pointwise solution of equations in $\mathbb{R}^{d}$ that depend only on the local characteristics $(b, c, F)(\omega, t)$. Ignoring the fact that it is generally a signed measure, the minimal martingale measure in the sense of Föllmer and Schweizer (1991), Schweizer (1995) constitutes a first example. The neutral pricing measure in Kallsen (2002) is of this type as well. Thirdly, the EMM leading to log-optimal portfolios shares this simple structure, which explains the often stated myopia of logarithmic utility (cf., Goll and Kallsen 2000). For continuous processes, there is in some sense only one "natural" local measure change, which is why these local approaches lead to the same equivalent martingale measure in this case.

\section{References}

Bellini, F., Frittelli, M.: On the existence of minimax martingale measures. Technical Report 14/2000, Università degli Studi di Milano - Bicocca, 2000

Bühlmann, H., Delbaen, F., Embrechts, P., Shiryaev, A.: No-arbitrage, change of measure and conditional Esscher transforms. CWI Quaterly 9, 291-317 (1996)

Chan, T.: Pricing contingent claims on stocks driven by Lévy processes. Ann. Appl. Prob. 9, 504-528 (1999)

Choulli, T., Krawczyk, L., Stricker, C.: $\mathcal{E}$-martingales and their applications in mathematical finance. Ann. Appl. Prob. 26, 853-876 (1998)

Cvitanić, J., Pham, H., Touzi, N.: Super-replication in stochastic volatility models under portfolio constraints. J. Appl. Prob. 36, 523-545 (1999)

Davis, M.: Option pricing in incomplete markets. In: Dempster, M., Pliska, S. (eds.), Mathematics of derivative securities, Cambridge: Cambridge University Press, 1997, pp. 216-226

Delbaen, F., Grandits, P., Rheinländer, T., Samperi, D., Schweizer, M., Stricker, C.: Exponential hedging and entropic penalties. Berlin: Preprint Technische Universität 2000

Delbaen, F., Haezendonck, J.: A martingale approach to premium calculation in an arbitrage free market. Insurance: Math. Econ. 8, 269-277 (1989)

Eberlein, E., Jacod, J.: On the range of option prices. Finance Stochast. 1, 131-140 (1997)

Eberlein, E., Keller, U.: Hyperbolic distributions in finance. Bernoulli 1, 281-299 (1995)

Esscher, F.: On the probability function in the collective theory of risk. Skand. Aktuarietidskrift 15, 175-195 (1932)

Foldes, L.: Conditions for optimality in the infinite-horizon portfolio-cum-saving problem with semimartingale investments. Stochast. Stochast. Reports 29, 133-170 (1990)

Föllmer, H., Schweizer, M.: Hedging of contingent claims under incomplete information. In: Davis, M.H.A., Elliott, R.J. (eds.) Applied stochastic analysis (Stochastics Monographs, vol. 5). London: Gordon \& Breach 1991, pp. 389-414

Frey, R., Sin, C.: Bounds on European option prices under stochastic volatility. Math. Finance 9(2), 97-116 (1999)

Frittelli, M.: The minimal entropy martingale measure and the valuation problem in incomplete markets. Math. Finance 10(1), 39-52 (2000)

Gerber, H., Shiu, E.: Option pricing by Esscher transforms. Transactions Soc. Actuaries 46, 99-191 (1994)

Gihman, I., Skorohod, A.: Stochastic differential equations. Berlin Heidelberg New York: Springer 1972

Goll, T., Kallsen, J.: Optimal portfolios for logarithmic utility. Stochast. Proc. Appl. 89, 31-48 (2000)

Goll, T., Rüschendorf, L.: Minimax and minimal distance martingale measures and their relationship to portfolio optimization. Finance Stochast. 5, 557-581 (2001) 
Grandits, P.: On martingale measures for stochastic processes with independent increments. Theory Prob. Appl. 44, 39-50 (1999a)

Grandits, P.: The $p$-optimal martingale measure and its asymptotic relation with the minimal-entropy martingale measure. Bernoulli 5, 225-247 (1999b)

Grigelionis, B.: The absolute continuity of measures that correspond to stochastic processes. Litovskiı Matematicheskiı̌ Sbornik 11, 783-794 (1971)

He, H., Pearson, N.: Consumption and portfolio policies with incomplete markets and short-sale constraints: The finite-dimensional case. Math. Finance 1(3), 1-10 (1991a)

He, H., N. Pearson.: Consumption and portfolio policies with incomplete markets and short-sale constraints: The infinite-dimensional case. J. Econ. Theory 54, 259-304 (1991b)

Jacod, J.: Calcul stochastique et problèmes de martingales (Lecture Notes in Mathematics, vol. 714). Berlin Heidelberg New York: Springer 1979

Jacod, J.: Intégrales stochastiques par rapport à une semi-martingale vectorielle et changements de filtration. In: Séminaire de Probabilités XIV, 1978/79. (Lecture Notes in Mathematics, vol. 784). Berlin Heidelberg New York: Springer 1980, pp. 161-172

Jacod, J., Mémin, J.: Caractéristiques locales et conditions de continuité absolue pour les semimartingales. Zeitschrift für Wahrscheinlichkeitstheorie und verwandte Gebiete 35, 1-37 (1976)

Jacod, J., Shiryaev, A.: Limit theorems for stochastic processes. Berlin Heidelberg New York: Springer 1987

Jerschow, M.: Uniform integrability of exponential martingales and SDEs. Stochast. Stochast. Reports 49, 139-147 (1994)

Kabanov, Y., Liptser, R., Shiryaev, A.: Absolute continuity and singularity of locally absolutely continuous probability distributions I. Math. USSR-Sbornik 35, 631-680 (1979)

Kabanov, Y., Liptser, R., Shiryaev, A.: Absolute continuity and singularity of locally absolutely continuous probability distributions II. Math. USSR-Sbornik 36, 31-58 (1980)

Kallsen, J.: Duality links between portfolio optimization and derivative pricing. Technical Report 40/1998, Mathematische Fakultät Universität Freiburg i. Br. 1998

Kallsen, J.: Optimal portfolios for exponential Lévy processes. Math. Meth. Oper. Res. 51, 357-374 (2000)

Kallsen, J.: Derivative pricing based on local utility maximization. Finance Stochast. 6, 115-140 (2002)

Kallsen, J.: Utility-based derivative pricing in incomplete markets. In: Geman, H., Madan, D., Pliska, S., Vorst, T. (eds.), Mathematical Finance - Bachelier Congress 2000, Berlin Heidelberg New York: Springer 2001, pp. 313-338

Kallsen, J., Shiryaev, A.: Time change representations of stochastic integrals. Theory Prob. Appl. 46 (2001) (forthcoming)

Karatzas, I., Kou, S.: On the pricing of contingent claims under constraints. Ann. Appl. Prob. 6, 321-369 (1996)

Karatzas, I., Lehoczky, J., Shreve, S., Xu, G.: Martingale and duality methods for utility maximization in an incomplete market. SIAM J. Control Opt. 29, 702-730 (1991)

Kazamaki, N.: On a problem of Girsanov. Tôhoku Math. J. 29, 597-600 (1977)

Kazamaki, N.: A note on exponential martingales. Tôhoku Math. J. 30, 367-371 (1978a)

Kazamaki, N.: A remark on a problem of Girsanov. In: Séminaire de Probabilités XII, 1976/77 (Lecture Notes in Mathematics vol. 649). Berlin Heidelberg New York: Springer 1978b, pp. 47-50

Kazamaki, N.: A sufficient condition for the uniform integrability of exponential martingales. Toyama University Mathematical Reports 2, 1-11 (1979)

Kazamaki, N.; Sekiguchi, T.: Remarks on a criterion for the uniform integrability of positive local martingales. Toyama University Mathematical Reports 5, 95-101 (1982)

Kazamaki, N., Sekiguchi, T.: Uniform integrability of continuous exponential martingales. Tôhoku Math. J. 35, 289-301 (1983)

Keller, U.: Realistic modelling of financial derivatives. Dissertation Universität Freiburg i. Br. 1997

Kramkov, D., Schachermayer, W.: The asymptotic elasticity of utility functions and optimal investment in incomplete markets. Ann. Appl. Prob. 9, 904-950 (1999)

Kramkov, D., Shiryaev, A.: Sufficient conditions of the uniform integrability of exponential martingales. In: European Congress of Mathematics, vol. I (Budapest, 1996), pp. 289-295. Basel: Birkhäuser 1998 
Lépingle, D., Mémin, J.: Intégrabilité uniforme et dans $L^{r}$ des martingales exponentielles. In: Séminaire de Probabilités. Rennes: Université de Rennes 1978a

Lépingle, D., Mémin, J.: Sur l'intégrabilité uniforme des martingales exponentielles. Zeitschrift für Wahrscheinlichkeitstheorie und verwandte Gebiete 42, 175-203 (1978b)

Liptser, R., Shiryaev, A.: On the absolute continuity of measures corresponding to processes of diffusion type relative to a Wiener measure. Mathematics of the USSR-Izvestiya 6, 839-882 (1972)

Madan, D., Milne, F.: Option pricing with V. G. martingale components. Math. Finance 1(4), 39-55 (1991)

Mémin, J.: (1978) Décompositions multiplicatives de semimartingales exponentielles et applications. In: Séminaire de Probabilités XII, 1976/77 (Lecture Notes in Mathematics, vol. 649). Berlin Heidelberg New York: Springer 1978, pp. 35-46

Mémin, J., Shiryaev, A.: Un critère prévisible pour l'uniforme intégrabilité des semimartingales exponentielles. In: Séminaire de Probabilités XIII, 1977/78 (Lecture Notes in Mathematics, vol. 721). Berlin Heidelberg New York: Springer 1979, pp. 147-161

Miyahara, Y.: Canonical martingale measures of incomplete assets markets. In: Watanabe, S., Fukushima, M., Prohorov, Y., Shiryaev, A. (eds.) Probability Theory and Mathematical Statistics: Proc. Seventh Japan-Russia Symposium, Tokyo 1995, pp. 343-352. World Scientific, Singapore 1996

Miyahara, Y.: Minimal entropy martingale measures of jump type price processes in incomplete assets markets. Asia-Pacific Financial Markets 6, 97-113 (1999)

Naik, V., Lee, M.: General equilibrium pricing of options on the market portfolio with discontinuous returns. Rev. Financial Stud. 3, 493-521 (1990)

Novikov, A.: On an identity for stochastic integrals. Theory Prob. Appl. 17, 717-720 (1972)

Novikov, A.: On discontinuous martingales. Theory Prob. Appl. 20, 11-26 (1975)

Novikov, A.: Conditions for absolute continuity of probability measures. Math. USSR-Sbornik 35, 697-707 (1979)

Novikov, A.: On conditions for uniform integrability of continuous non-negative martingales. Theory Prob. Appl. 24, 820-824 (1980)

Okada, T.: A criterion for uniform integrability of exponential martingales. Tôhoku Math. J. 34, 495-498 (1982)

Revuz, D., Yor, M.: Continuous Martingales and Brownian Motion, 3rd ed. Berlin Heidelberg New York: Springer 1999

Rockafellar, T.: Convex Analysis. Princeton University Press, Princeton 1970

Schachermayer, W.: How potential investments may change the optimal portfolio for the exponential utility. Preprint 2000

Schachermayer, W.: Optimal investment in incomplete markets when wealth may become negative. Ann. Appl. Prob. 11, 694-734 (2001)

Schäl, M.: On quadratic cost criteria for options hedging. Math. Opera. Res. 19, 121-131 (1994)

Schweizer, M.: On the minimal martingale measure and the Föllmer-Schweizer decomposition. Stochast. Anal. Appl. 13, 573-599 (1995)

Schweizer, M.: Approximation pricing and the variance-optimal martingale measure. Ann. Prob. 24, 206-236 (1996)

Shiryaev, A. Essentials of Stochastic Finance. Singapore: World Scientific 1999

Stummer, W.: The Novikov and entropy conditions of multidimensional diffusion processes with singular drift. Prob. Theory Rel. Fields 97, 515-542 (1993)

Witting, H.: Mathematische Statistik I. Stuttgart: Teubner 1985

Wolfe, S.: On moments of infinitely divisible distribution functions. Ann. Math. Stat. 42, 2036-2043 (1971)

Xia, J., Yan, J.: Martingale measure method for expected utility maximization and valuation in incomplete markets. Preprint 2000

Yan, J.: Criteria for the uniform integrability of exponential martingales. Acta Math. Sinica 23, 293-300 (1980)

Yan, J.: À propos de l'inteǵrabilité uniforme des martingales exponentielles. In: Séminaire de Probabilités XVI (Lecture Notes in Mathematics, vol. 920). Berlin Heidelberg New York: Springer 1982a, pp. 338-347

Yan, J.: Uniform and $L^{r}$-integrability of exponential martingales. Chin. Ann. Math. 3, 285-292 (1982b) 